\title{
Instantaneous Mutual Information and Eigen-Channels in MIMO Mobile Rayleigh Fading
}

\author{
Shuangquan Wang ${ }^{\dagger}$, and Ali Abdi ${ }^{\ddagger}$, Senior Member, IEEE
}

\begin{abstract}
In this paper, we study two important metrics in multiple-input multiple-output (MIMO) time-varying Rayleigh flat fading channels. One is the eigen-channel, and the other is the instantaneous mutual information (IMI). Their secondorder statistics, such as the correlation coefficient, level crossing rate (LCR), and average fade/outage duration, are investigated, assuming a general nonisotropic scattering environment. Exact closed-form expressions are derived and Monte Carlo simulations are provided to verify the accuracy of the analytical results. For the eigen-channels, we found they tend to be spatio-temporally uncorrelated in large MIMO systems. For the IMI, the results show that its correlation coefficient can be well approximated by the squared amplitude of the correlation coefficient of the channel, under certain conditions. Moreover, we also found the LCR of IMI is much more sensitive to the scattering environment than that of each eigen-channel.
\end{abstract}

Index Terms-Eigen-channels, Instantaneous Mutual Information, Autocorrelation Function, Correlation Coefficient, Level Crossing Rate, Average Fade/Outage Duration, and MultipleInput Multiple-Output (MIMO).

\section{INTRODUCTION}

$\mathbf{T}$ HE utilization of antenna arrays at the base station (BS) and the mobile station (MS) in a wireless communication system increases the capacity linearly with $\min \left(N_{T}, N_{R}\right)$, under certain conditions, where $N_{T}$ and $N_{R}$ are numbers of transmit and receive antenna elements, respectively, provided that the environment is sufficiently rich in multi-path components [1][2]. This is due to the fact that a multipleinput multiple-output (MIMO) channel can be decomposed to several parallel single-input single-output (SISO) channels, called eigen-channels, via singular value decomposition (SVD) [2]-[9].

For a SISO channel, or any subchannel ${ }^{1}$ of a MIMO system, there are numerous studies on key second-order statistics such as correlation, level crossing rate (LCR), and average fade duration (AFD) [10]-[14]. However, to the best of our

Manuscript received February 22, 2010; revised May 06, 2011; Accepted June 26, 2011.

This paper was presented in part at the 40th Ann. Conf. Info. Sci. Sys., Princeton, NJ, 2006, and IEEE Globecom, San Francisco, CA, 2006.

${ }^{\dagger} \mathrm{S}$. Wang was with the Department of Electrical and Computer Engineering, New Jersey Institute of Technology, Newark, NJ 07102, USA. He is now with Broadcom Corporation, Matawan, NJ 07747, USA. (e-mail:sw27@njit.edu).

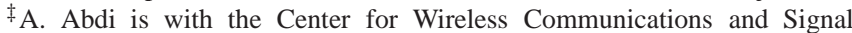
Processing Research (CWCSPR), Department of Electrical and Computer Engineering, New Jersey Institute of Technology, Newark, NJ 07102, USA (e-mail:ali.abdi@njit.edu).

Communicated by Prof. Taricco, Associate Editor for Communications.

Color versions of one or more of the figures in this paper are available online at http://ieeexplore.ieee.org.

${ }^{1}$ In this paper, each subchannel represents the radio link between each transmit/receive pair of antennas. knowledge, no such study on the eigen-channels of a MIMO system is reported in the literature, possibly due to the lack of knowledge regarding the joint probability density function (PDF) of eigen-channels.

Regarding another important quantity, the instantaneous mutual information (IMI), only some first-order statistics such as the mean, variance, outage probability and PDF are studied [9][15]-[18]. Clearly, those statistics do not show the dynamic temporal behavior, such as correlations, LCR and average outage durations (AOD) of the IMI in time-varying fading channels. It is known that IMI can be feedbacked to the rate scheduler in multi-user communication environments, to increase the system throughput [16], where only the perfect feedback is considered. However, it is hard to obtain perfect feedback in practice due to the time-varying nature of the channel, which makes the feedbacked IMI outdated. In this case, the temporal correlation of IMI can be used to analyze the scheduling performance with outdated IMI feedbacks. Furthermore, one can improve the rate scheduling algorithm by exploring the temporal correlation of IMI.

Several second-order statistics such as the correlation coefficient, LCR and AOD of IMI in single-input single-output (SISO) systems are reported in [19]. However, there are a limited number of results for a general MIMO channel. In [20], some simulation results regarding the correlation coefficient, LCR and AOD are reported, without analytical derivations. In [21], lower and upper bounds, as well as some approximations for the correlation coefficient of IMI are derived, without exact results at high SNR. A large gap between the lower and upper bounds and large approximation errors are observed in [21, Figs. 2, 5].

In this paper, we study the general MIMO case using the joint PDF of the eigenvalues [22]. Specifically, a number of second-order statistics such as the autocorrelation function (ACF), the correlation coefficient, LCR and AFD/AOD ${ }^{2}$ of the eigen-channels and the IMI are studied in MIMO timevarying Rayleigh flat fading channels. We assume all the subchannels are spatially independent and identically distributed (i.i.d.), with the same temporal correlation coefficient, considering general nonisotropic scattering propagation environments. Closed-form expressions are derived, and Monte Carlo simulations are provided to verify the accuracy of our closed-form expressions. The simulation and analytical results show that the eigen-channels tend to be spatio-temporally uncorrelated in large MIMO systems, and the correlation

\footnotetext{
${ }^{2}$ Note that AFD is used for eigen-channels, whereas AOD is used for MIMO IMI
} 
coefficient of the IMI can be well approximated by the squared amplitude of the correlation coefficient of the channel if $\left|N_{T}-N_{R}\right|$ is much larger than $\min \left(N_{T}, N_{R}\right)$. In addition, we also observed that the LCR of IMI is much more sensitive to the scattering environment than that of each eigen-channel.

The rest of this paper is organized as follows. Section II introduces the channel model, as well as the angle-ofarrival (AoA) model. Eigen-channels of a MIMO system are discussed in Section III, where Subsection III-A is devoted to the derivation of the normalized ACF (NACF) and the correlation coefficient of eigen-channels of a MIMO system, whereas Subsection III-B focuses on the LCR and AFD of the eigen-channels. The MIMO IMI is investigated in Section IV, in which Subsection IV-A addresses the NACF and the correlation coefficient of the MIMO IMI as well as their low- and high-SNR approximations, whereas Subsection IV-B studies the LCR and AOD of the MIMO IMI using the well-known Gaussian approximation. Numerical results and discussions are presented in Section V, and concluding remarks are given in Section VI.

Notation:.$^{\dagger}$ is reserved for matrix Hermitian, $\cdot{ }^{\star}$ for complex conjugate, $\jmath$ for $\sqrt{-1}, \mathbb{E}[\cdot]$ for mathematical expectation, $\mathbf{I}_{m}$ for the $m \times m$ identity matrix, $\|\cdot\|_{F}$ for the Frobenius norm, and $f^{2}(x)$ for $[f(x)]^{2}$. Finally, $t \in[m, n]$ implies that $t, m$ and $n$ are integers such that $m \leq t \leq n$ with $m \leq n$.

\section{Channel Model}

In this paper, an $N_{R} \times N_{T}$ MIMO time-varying Rayleigh flat fading channel is considered. Similar to [15], we consider a piecewise constant approximation for the continuous-time MIMO fading channel matrix coefficient $\mathbf{H}(t)$, represented by $\left\{\mathbf{H}\left(l T_{s}\right)\right\}_{l=1}^{L}$, where $T_{s}$ is the symbol duration and $L$ is the number of samples. In the sequel, we drop $T_{s}$ to simplify the notation. In the $l^{\text {th }}$ symbol duration, the matrix of the channel coefficients is given by

$$
\mathbf{H}(l)=\left[\begin{array}{ccc}
h_{1,1}(l) & \cdots & h_{1, N_{T}}(l) \\
\vdots & \ddots & \vdots \\
h_{N_{R}, 1}(l) & \cdots & h_{N_{R}, N_{T}}(l)
\end{array}\right], l \in[1, L] .
$$

We assume all the $N_{T} N_{R}$ subchannels $\left\{h_{n_{r}, n_{t}}(l), l \in[1, L]\right\}_{\left(n_{r}=1, n_{t}=1\right)}^{\left(N_{R}, N_{T}\right)}$ are i.i.d., with the same temporal correlation coefficient, i.e.,

$$
\mathbb{E}\left[h_{m n}(l) h_{p q}^{\star}(l-i)\right]=\delta_{m, p} \delta_{n, q} \rho_{h}(i),
$$

where the Kronecker delta $\delta_{m, p}$ is 1 or 0 when $m=p$ or $m \neq p$, respectively, and $\rho_{h}(i)$ is defined and derived at the end of this section, eq. (4).

In flat Rayleigh fading channels, each $h_{n_{r}, n_{t}}(l), l \in[1, L]$, is a zero-mean complex Gaussian random process. In the $l^{\text {th }}$ interval, $h_{n_{r}, n_{t}}(l)$ can be represented as [13]

$$
\begin{aligned}
h_{n_{r}, n_{t}}(l) & =h_{n_{r}, n_{t}}^{I}(l)+\jmath h_{n_{r}, n_{t}}^{Q}(l), \\
& =\alpha_{n_{r}, n_{t}}(l) \exp \left[-\jmath \Phi_{n_{r}, n_{t}}(l)\right],
\end{aligned}
$$

where the zero-mean real Gaussian random processes $h_{n_{r}, n_{t}}^{I}(l)$ and $h_{n_{r}, n_{t}}^{Q}(l)$ are the real and imaginary parts of $h_{n_{r}, n_{t}}(l)$, respectively. $\alpha_{n_{r}, n_{t}}(l)$ is the envelope of $h_{n_{r}, n_{t}}(l)$ and $\Phi_{n_{r}, n_{t}}(l)$ is the phase of $h_{n_{r}, n_{t}}(l)$. For each $l, \alpha_{n_{r}, n_{t}}(l)$

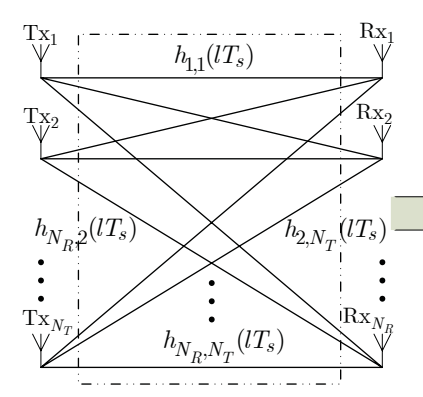

(a) An $N_{R} \times N_{T}$ MIMO Channel

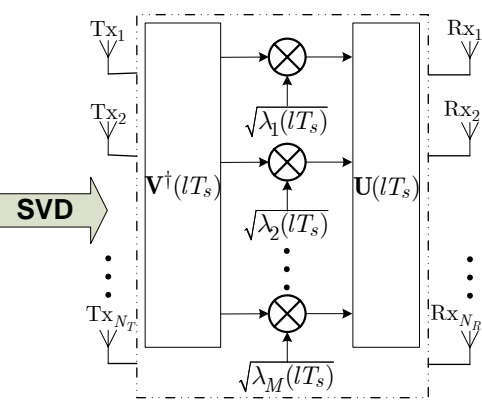

(b) $M$ Parallel SISO Channels
Figure 1. (a) A MIMO channel with $N_{T}$ transmit and $N_{R}$ receive antennas; (b) The equivalent $M$ parallel SISO channel representation.

has a Rayleigh distribution and $\Phi_{n_{r}, n_{t}}(l)$ is distributed uniformly over $[-\pi, \pi)$. Without loss of generality, we assume each subchannel has unit power, i.e., $\mathbb{E}\left[\alpha_{n_{r}, n_{t}}^{2}(l)\right]=1$.

Using empirically-verified [13] multiple von Mises PDF's $[19,(4)]$ for the AoA at the receiver in nonisotropic scattering environments, shown as Fig. 1 of [19], the channel correlation coefficient of $h_{n_{r}, n_{t}}(l), \forall n_{r}, n_{t}$, is given by [19, (7)]

$$
\begin{aligned}
& \rho_{h}(i)=\mathbb{E}\left[h_{n_{r}, n_{t}}(l) h_{n_{r}, n_{t}}^{\star}(l-i)\right], \\
& =\sum_{n=1}^{N} P_{n} \frac{I_{0}\left(\sqrt{\kappa_{n}^{2}-4 \pi^{2} f_{D}^{2} i^{2} T_{s}^{2}+\jmath 4 \pi \kappa_{n} f_{D} i T_{s} \cos \theta_{n}}\right)}{I_{0}\left(\kappa_{n}\right)},
\end{aligned}
$$

where $I_{k}(z)=\frac{1}{\pi} \int_{0}^{\pi} e^{z \cos w} \cos (k w) \mathrm{d} w$ is the $k^{\text {th }}$ order modified Bessel function of the first kind, $\theta_{n}$ is the mean AoA of the $n^{\text {th }}$ cluster of scatterers, $\kappa_{n}$ controls the width of the $n^{\text {th }}$ cluster of scatterers, $P_{n}$ represents the contribution of the $n^{\text {th }}$ cluster of scatterers such that $\sum_{n=1}^{K} P_{n}=1,0<P_{n} \leq 1, K$ is the number of clusters of scatterers, and $f_{D}$ is the maximum Doppler frequency. When $\kappa_{n}=0, \forall n$, which corresponds to isotropic scattering, (4) reduces to $\rho_{h}(i)=I_{0}\left(\jmath 2 \pi f_{D} i T_{s}\right)=$ $J_{0}\left(2 \pi f_{D} i T_{s}\right)$, which is the Clarke's correlation model. In (4), $I_{0}\left(\sqrt{\kappa_{n}^{2}-4 \pi^{2} f_{D}^{2} i^{2} T_{s}^{2}+\jmath 4 \pi \kappa_{n} f_{D} i T_{s} \cos \theta_{n}}\right) / I_{0}\left(\kappa_{n}\right)$ is the correlation of the $n^{\text {th }}$ cluster, and the overall channel correlation is a weighted sum of correlations from all clusters.

\section{EIgEN-ChANNELS IN MIMO Systems}

We set $M=\min \left(N_{T}, N_{R}\right)$ and $N=\max \left(N_{T}, N_{R}\right)$. Based on singular value decomposition (SVD) [2]-[9], $\mathbf{H}(l)$ in (1) can be diagonalized in the following form

$$
\mathbf{H}(l)=\mathbf{U}(l) \mathbf{S}(l) \mathbf{V}^{\dagger}(l),
$$

where $\mathbf{V}(l)$, whose dimension is $N_{T} \times M$, satisfies $\mathbf{V}^{\dagger}(l) \mathbf{V}(l)=\mathbf{I}_{M}, \mathbf{U}(l)$, which is $N_{R} \times M$, satisfies $\mathbf{U}^{\dagger}(l) \mathbf{U}(l)=\mathbf{I}_{M}$, and $\mathbf{S}(l)$ is a diagonal matrix, given by $\mathbf{S}(l)=\operatorname{diag}\left[s_{1}(l), \cdots, s_{M}(l)\right]$, in which $s_{m}(l), m \in[1, M]$ is the $m^{\text {th }}$ non-zero singular value of $\mathbf{H}(l)$.

We define $\lambda_{m}(l)=s_{m}^{2}(l), \forall m$. Therefore $\lambda_{m}(l)$ is the $m^{\text {th }}$ non-zero eigenvalue of $\mathbf{H}(l) \mathbf{H}^{\dagger}(l)$. We further consider $\left\{\lambda_{m}(l)\right\}_{m=1}^{M}$ as unordered non-zero eigenvalues of $\mathbf{H}(l) \mathbf{H}^{\dagger}(l)$. Therefore, the MIMO channel $\mathbf{H}(l)$ is decomposed to $M$ identically distributed eigen-channels, $\left\{\lambda_{m}(l), l \in[1, L]\right\}_{m=1}^{M}$, by SVD, as shown in Fig. 1. For $M=1$, there is only 
one eigen-channel, which corresponds to the maximal ratio transmitter (MRT) if $N_{R}=1$, or the maximal ratio combiner (MRC) if $N_{T}=1$. In each case, we have $N$ i.i.d complex Gaussian branches.

Since all the eigen-channels have identical statistics, we only study one of them and denote it as $\lambda(l), l \in[1, L]$. To simplify the notation, we use $X$ and $Y$ to denote $\lambda(l)$ and $\lambda(l-i)$, respectively. The joint PDF of $X$ and $Y$ is given in (4.11) of [22],

$$
\begin{aligned}
& p(x, y)= \frac{(x y)^{\frac{\nu}{2}} e^{-\frac{x+y}{1-\varrho_{i}^{2}}} I_{\nu}\left(\frac{2 \varrho_{i} \sqrt{x y}}{1-\varrho_{i}^{2}}\right)}{M^{2}\left(1-\varrho_{i}^{2}\right) \varrho_{i}^{\nu}} \sum_{k=0}^{M-1} \frac{k ! L_{k}^{\nu}(x) L_{k}^{\nu}(y)}{(k+\nu) ! \varrho_{i}^{2 k}} \\
&+ \frac{(x y)^{\nu} e^{-(x+y)}}{M^{2}} \sum_{0 \leq k<l}^{M-1}\left\{\frac{k ! l !}{(k+\nu) !(l+\nu) !}\right. \\
& \times\left[\left[L_{k}^{\nu}(x) L_{l}^{\nu}(y)\right]^{2}+\left[L_{l}^{\nu}(x) L_{k}^{\nu}(y)\right]^{2}\right. \\
&\left.\left.-\left(\varrho_{i}^{2(l-k)}+\varrho_{i}^{2(k-l)}\right) L_{k}^{\nu}(x) L_{l}^{\nu}(x) L_{k}^{\nu}(y) L_{l}^{\nu}(y)\right]\right\}
\end{aligned}
$$

where $L_{n}^{\alpha}(x)=\frac{1}{n !} e^{x} x^{-\alpha} \frac{\mathrm{d}^{n}}{\mathrm{~d} x^{n}}\left(e^{-x} x^{n+\alpha}\right)$ is the associated Laguerre polynomial of order $n$ [23, pp. 1061, 8.970.1], $\nu=N-M$, and $\varrho_{i}=\left|\rho_{h}(i)\right|$, where $\rho_{h}(i)$ is given in (4). Lemma 7 shows that (6) can be rewritten as the product of the two marginal PDFs plus a cross term. The cross term indicates that two eigen-values at different time indices are dependent.

The joint PDF in (6) is very general and includes many existing PDF's as special cases [22].

- By integration over $y$, (6) reduces to the marginal PDF

$$
p(x)=\frac{1}{M} \sum_{m=0}^{M-1} \frac{m !}{(m+\nu) !}\left[L_{m}^{\nu}(x)\right]^{2} x^{\nu} e^{-x},
$$

which is the same as the PDF presented in [2]. When $M=1$, (7) further reduces to

$$
p(x)=\frac{1}{(N-1) !} x^{N-1} e^{-x}
$$

which is the $\chi^{2}$ distribution with $2 N$ degrees of freedom [24, (2.32)], used for characterizing the PDF of outputs of MRT or MRC [25].

- With $M=1$, (6) reduces to

$$
p(x, y)=\frac{(x y)^{\frac{N-1}{2}} \exp \left(-\frac{x+y}{1-\varrho_{i}^{2}}\right) I_{N-1}\left(\frac{2 \varrho_{i} \sqrt{x y}}{1-\varrho_{i}^{2}}\right)}{(N-1) !\left(1-\varrho_{i}^{2}\right) \varrho_{i}^{N-1}},
$$

which is the joint PDF of outputs of MRT or MRC at the $l^{\text {th }}$ and $(l-i)^{\text {th }}$ symbol durations [26]. It includes (3.14) of [24] as a special case ${ }^{3}$. Furthermore, when $N=1$, i.e., a SISO channel, (9) simplifies to

$$
p(x, y)=\frac{1}{1-\varrho_{i}^{2}} \exp \left(-\frac{x+y}{1-\varrho_{i}^{2}}\right) I_{0}\left(\frac{2 \varrho_{i} \sqrt{x y}}{1-\varrho_{i}^{2}}\right),
$$

which is identical to (8-103) [27, pp. 163], after a oneto-one nonlinear mapping.

${ }^{3}$ Eq. (3.14) in [24] is developed for real uncorrelated Gaussian random variables.
In the following subsections, we study the normalized correlation and correlation coefficient of any two eigen-channels, defined by, respectively,

$$
\widetilde{r}_{m, n}(i)=\frac{\mathbb{E}\left[\lambda_{m}(l) \lambda_{n}(l-i)\right]}{\sqrt{\mathbb{E}\left[\lambda_{m}^{2}(l)\right]} \sqrt{\mathbb{E}\left[\lambda_{n}^{2}(l-i)\right]}},
$$

and

$$
\begin{gathered}
\rho_{m, n}(i)=\frac{\mathbb{E}\left[\lambda_{m}(l) \lambda_{n}(l-i)\right]-\mathbb{E}\left[\lambda_{m}(l)\right] \mathbb{E}\left[\lambda_{n}(l-i)\right]}{\sqrt{\mathbb{E}\left[\lambda_{m}^{2}(l)\right]-\left\{\mathbb{E}\left[\lambda_{m}(l)\right]\right\}^{2}}} \\
/ \sqrt{\mathbb{E}\left[\lambda_{n}^{2}(l-i)\right]-\left\{\mathbb{E}\left[\lambda_{n}(l-i)\right]\right\}^{2}}, \quad
\end{gathered}
$$

\section{A. Normalized Correlation and Correlation Coefficient of Eigen-Channels}

To derive the normalized correlation and correlation coefficient between any two eigen-channels, we need the following lemmas.

Lemma 1: The first and second moments of the $m^{\text {th }}$ eigenchannel are respectively given by

$$
\begin{aligned}
& \mathbb{E}\left[\lambda_{m}(l)\right]=N, \\
& \mathbb{E}\left[\lambda_{m}^{2}(l)\right]=N(N+M) .
\end{aligned}
$$

Proof: See Appendix A.

Lemma 2: The autocorrelation of the $m^{\text {th }}$ eigen-channel, defined as $r_{m, m}(i)=\mathbb{E}\left[\lambda_{m}(l) \lambda_{m}(l-i)\right]$, is given by

$$
r_{m, m}(i)=N^{2}+\frac{N \varrho_{i}^{2}}{M}, \quad i \neq 0 .
$$

Proof: See Appendix B

Lemma 3: The cross-correlation between the $m^{\text {th }}$ and $n^{\text {th }}$ eigen-channels, defined as $r_{m, n}(i)=\mathbb{E}\left[\lambda_{m}(l) \lambda_{n}(l-i)\right]$, is given by

$$
r_{m, n}(i)=\left\{\begin{array}{ll}
N^{2}-N, & i=0, \\
N^{2}+\frac{N \varrho_{i}^{2}}{M}, & i \neq 0,
\end{array} n \neq m .\right.
$$

Proof: See Appendix C.

Based on Lemmas 1-3, we obtain the closed-form expressions for (11) and (12), which are given in the following theorem.

Theorem 1: The normalized cross-correlation and the correlation coefficient between $m^{\text {th }}$ and $n^{\text {th }}$ eigen-channels, defined in (11) and (12), are respectively given by

$$
\widetilde{r}_{m, n}(i)= \begin{cases}\frac{N+M-(M+1)\left(1-\delta_{m, n}\right)}{M N+\varrho_{i}^{2}}, & i=0, \\ \frac{M+M}{M N+M^{2}}, & i \neq 0,\end{cases}
$$

and

$$
\rho_{m, n}(i)= \begin{cases}1-\frac{M+1}{M}\left(1-\delta_{m, n}\right), & i=0, \\ \frac{\varrho_{i}^{2}}{M^{2}}, & i \neq 0 .\end{cases}
$$

Proof: Lemma 1 shows that the eigen-channel is stationary in the wide sense. Moreover, all the eigen-channels have the same statistics, therefore we have $\mathbb{E}\left[\lambda_{n}(l-i)\right]=$ $\mathbb{E}\left[\lambda_{m}(l)\right]$ and $\mathbb{E}\left[\lambda_{n}^{2}(l-i)\right]=\mathbb{E}\left[\lambda_{m}^{2}(l)\right], \forall m, n \in[1, M]$ and $\forall l, i$. By plugging (14)-(15) into (11), we obtain (17). Finally, substitution of (13)-(15) into (12) results in (18). 
According to (17) and (18), one can see correlations at non-zero lags do not depend on the channel indices $m$ and $n$. Moreover, at lag zero, correlations have two values only, one for $m=n$ and the other for $m \neq n$. We also have the following observations with regard to (17) and (18).

- If $M$ is greater than 1 , the normalized correlation and the correlation coefficient are not continuous at $i=0$, as $\tilde{r}_{m, n}(1)$ and $\rho_{m, n}(1)$ do not converge to $\tilde{r}_{m, n}(0)=$ $\rho_{m, n}(0)=1$ as $T_{s} \rightarrow 0, \forall m, n$. This is because the eigenvalues are not ordered.

- If $M$ is large, all the $M$ eigen-channels tend to be spatiotemporally uncorrelated, due to

$$
\lim _{M \rightarrow \infty} \rho_{m, n}(i)=\delta_{m, n} \delta_{i, 0} .
$$

As an example, with isotropic scattering, (17) and (18), respectively, reduce to

$$
\widetilde{r}_{m, n}(i)= \begin{cases}\frac{N+M-(M+1)\left(1-\delta_{m, n}\right)}{N+M}, & i=0, \\ \frac{M N+J_{0}^{2}\left(2 \pi f_{D} i T_{s}\right)}{M N+M^{2}}, & i \neq 0,\end{cases}
$$

and

$$
\rho_{m, n}(i)= \begin{cases}1-\frac{M+1}{M}\left(1-\delta_{m, n}\right), & i=0, \\ \frac{J_{0}^{2}\left(2 \pi f_{D} i T_{s}\right)}{M^{2}}, & i \neq 0,\end{cases}
$$

\section{B. LCR and AFD of an Eigen-Channel}

In this subsection, we calculate the LCR and AFD of an eigen-channel at a given level. To simplify the notation, the eigen-channel index $m$ is dropped in this subsection, as the derived LCR and AFD results hold for any eigen-channel.

1) LCR of an Eigen-Channel: Similar to the calculation of zero crossing rate in discrete time [28, Ch. 4], we define the binary sequence $\left\{Z_{l}\right\}_{l=1}^{L}$, based on the eigen-channel samples $\{\lambda(l)\}_{l=1}^{L}$, as

$$
Z_{l}= \begin{cases}1, & \text { if } \lambda(l) \geq \lambda_{\mathrm{th}}, \\ 0, & \text { if } \lambda(l)<\lambda_{\mathrm{th}}\end{cases}
$$

where $\lambda_{\text {th }}$ is a fixed threshold. The number of crossings of $\{\lambda(l)\}_{l=1}^{L}$ with $\lambda_{\text {th }}$, within the time interval $T_{s} \leq t \leq L T_{s}$, denoted by $D_{\lambda_{\text {th }}}$, can be defined in terms of $\left\{Z_{l}\right\}_{l=1}^{L}[28$, (4.1)]

$$
D_{\lambda_{\mathrm{th}}}=\sum_{l=2}^{L}\left(Z_{l}-Z_{l-1}\right)^{2}
$$

which includes both up- and down-crossings.

After some simple manipulations, the expected crossing rate at the level $\lambda_{\text {th }}$ can be written as

$$
\frac{\mathbb{E}\left[D_{\lambda_{\mathrm{th}}}\right]}{(L-1) T_{s}}=\frac{2 P_{r}\left\{Z_{l}=1\right\}-2 P_{r}\left\{Z_{l}=1, Z_{l-1}=1\right\}}{T_{s}},
$$

where $P_{r}\{\cdot\}$ is the probability of an event. Therefore, the expected down crossing rate at $\lambda_{\text {th }}$, denoted by $N_{\lambda}\left(\lambda_{\text {th }}\right)$, is half of (24), given by

$$
N_{\lambda}\left(\lambda_{\mathrm{th}}\right)=\frac{\phi_{\lambda}\left(\lambda_{\mathrm{th}}\right)-\varphi_{\lambda}\left(\lambda_{\mathrm{th}}\right)}{T_{s}},
$$

where $\phi_{\lambda}\left(\lambda_{\mathrm{th}}\right)=P_{r}\left\{Z_{l}=1\right\}$ and $\varphi_{\lambda}\left(\lambda_{\mathrm{th}}\right)=P_{r}\left\{Z_{l}=\right.$ $\left.1, Z_{l-1}=1\right\}$. Analytical expressions for $\phi_{\lambda}\left(\lambda_{\mathrm{th}}\right)$ and $\varphi_{\lambda}\left(\lambda_{\mathrm{th}}\right)$ are stated in the following theorem.
Theorem 2: For a given threshold $\lambda_{\text {th }}, \phi_{\lambda}\left(\lambda_{\text {th }}\right)$ and $\varphi_{\lambda}\left(\lambda_{\text {th }}\right)$ are, respectively, given by

$$
\phi_{\lambda}\left(\lambda_{\mathrm{th}}\right)=\frac{1}{M} \sum_{m=0}^{M-1} \sum_{p=0}^{m} \sum_{q=0}^{m} \frac{m !\left(\begin{array}{c}
m+\nu \\
m-p
\end{array}\right)\left(\begin{array}{c}
m+\nu \\
m-q
\end{array}\right) \Gamma\left(p+q+\nu+1, \lambda_{\mathrm{th}}\right)}{(m+\nu) ! p ! q !(-1)^{p+q}},
$$

and

$$
\begin{gathered}
\varphi_{\lambda}\left(\lambda_{\mathrm{th}}\right)=\phi_{\lambda}^{2}\left(\lambda_{\mathrm{th}}\right)+\frac{1}{M^{2}} \sum_{j=M}^{\infty} \sum_{k=0}^{M-1}\left\{\frac{j ! k ! \varrho_{1}^{2(j-k)}}{(j+\nu) !(k+\nu) !}\right. \\
\left.\times\left[\sum_{p=0}^{j} \sum_{q=0}^{k} \frac{\left(\begin{array}{c}
j+\nu \\
j-p
\end{array}\right)\left(\begin{array}{c}
k+\nu \\
k-q
\end{array}\right)}{p ! q !(-1)^{p+q}} \Gamma\left(p+q+\nu+1, \lambda_{\mathrm{th}}\right)\right]^{2}\right\},
\end{gathered}
$$

where $\Gamma(a, z)=\int_{z}^{\infty} t^{a-1} e^{-t} \mathrm{~d} t$ [23, pp. 949, 8.350.2] is the upper incomplete gamma function, $\left(\begin{array}{l}n \\ k\end{array}\right)$ is the binomial coefficient, given by $\frac{n !}{k !(n-k) !}$, and $\varrho_{1}=\left|\rho_{h}(1)\right|$, defined before, i.e.,

$$
\varrho_{1}=\left|\sum_{n=1}^{N} P_{n} \frac{I_{0}\left(\sqrt{\kappa_{n}^{2}-4 \pi^{2} f_{D}^{2} T_{s}^{2}+\jmath 4 \pi \kappa_{n} f_{D} T_{s} \cos \theta_{n}}\right)}{I_{0}\left(\kappa_{n}\right)}\right| .
$$

Proof: $L_{n}^{\nu}(x)$ is a polynomial of order $n$, and can be represented as [23, pp. 1061, 8.970.1]

$$
L_{n}^{\nu}(x)=\sum_{k=0}^{n}\left(\begin{array}{l}
n+\nu \\
n-k
\end{array}\right) \frac{(-x)^{k}}{k !} .
$$

By plugging (29) into (7), the univariate PDF of an eigenchannel, and integrating over $x$ from $\lambda_{\text {th }}$ to $\infty$, we obtain (26). Similarly, substitution of (29) into (64), the bivariate PDF of an eigen-channel, and integration over $x$ from $\lambda_{\text {th }}$ to $\infty$ results in (27).

By plugging (26) and (27) into (25), we obtain the expected crossing rate at the level $\lambda_{\text {th }}$.

2) AFD of an Eigen-Channel: The cumulative distribution function (CDF) of $\lambda(l), \forall l$, is obtained as

$$
F_{\lambda}\left(\lambda_{\text {th }}\right)=P_{r}\left\{X \leq \lambda_{\text {th }}\right\}=1-\phi_{\lambda}\left(\lambda_{\text {th }}\right),
$$

where $\phi_{\lambda}\left(\lambda_{\text {th }}\right)$ is given in (26).

The AFD of the eigen-channel $\{\lambda(l)\}_{l=1}^{L}$ is therefore given by

$$
\bar{t}_{\lambda}\left(\lambda_{\mathrm{th}}\right)=\frac{F_{\lambda}\left(\lambda_{\mathrm{th}}\right)}{N_{\lambda}\left(\lambda_{\mathrm{th}}\right)}=\frac{\left[1-\phi_{\lambda}\left(\lambda_{\mathrm{th}}\right)\right] T_{s}}{\phi_{\lambda}\left(\lambda_{\mathrm{th}}\right)-\varphi_{\lambda}\left(\lambda_{\mathrm{th}}\right)},
$$

where $\phi_{\lambda}\left(\lambda_{\text {th }}\right)$ and $\varphi_{\lambda}\left(\lambda_{\text {th }}\right)$ are given in (26) and (27), respectively.

\section{MIMO IMI}

In this section, the NACF, the correlation coefficient, LCR and AOD of IMI in a MIMO system are investigated in detail. In the presence of the additive white Gaussian noise, if perfect channel state information $\{\mathbf{H}(l)\}_{l=1}^{L}$, is available at the receiver only, the ergodic channel capacity is given by [2][9]

$$
C=\mathbb{E}\left[\ln \operatorname{det}\left(\mathbf{I}_{N_{R}}+\frac{\eta}{N_{T}} \mathbf{H}_{l} \mathbf{H}_{l}^{\dagger}\right)\right],
$$

in nats $/ \mathrm{s} / \mathrm{Hz}$, where $\eta$ is the average SNR at each receive antenna, and $\mathbf{H}_{l}$ denotes $\mathbf{H}(l)$. 
In the above equation, at any given time index $l$, $\ln \operatorname{det}\left(\mathbf{I}_{N_{R}}+\frac{\eta}{N_{T}} \mathbf{H}_{l} \mathbf{H}_{l}^{\dagger}\right)$ is a random variable as it depends on the random channel matrix $\mathbf{H}_{l}$. Therefore

$$
\mathcal{I}_{l}=\ln \operatorname{det}\left(\mathbf{I}_{N_{R}}+\frac{\eta}{N_{T}} \mathbf{H}_{l} \mathbf{H}_{l}^{\dagger}\right), \quad l=1,2, \cdots,
$$

is a discrete-time random process with the ergodic capacity as its mean.

By plugging (5) into (33), we can express the IMI in terms of $M$ eigenvalues as

$$
\mathcal{I}_{l}=\sum_{m=1}^{M} \ln \left(1+\frac{\eta}{N_{T}} \lambda_{m}(l)\right), \quad l=1,2, \cdots .
$$

\section{A. NACF and Correlation Coefficient of MIMO IMI}

In this subsection, we derive exact closed-form expression fors the NACF and the correlation coefficient of MIMO IMI, and their approximations at low- and high-SNR regimes, using the following lemmas.

Lemma 4: The mean and second moment of $\mathcal{I}_{l}$ are respectively given by (35) and (36)

$$
\begin{aligned}
& \mathbb{E}\left[\mathcal{I}_{l}\right]=\sum_{m=0}^{M-1} \sum_{p=0}^{m} \sum_{q=0}^{m} \frac{m !\left(\begin{array}{l}
m+\nu \\
m-p
\end{array}\right)\left(\begin{array}{l}
m+\nu \\
m-q
\end{array}\right)}{(m+\nu) ! p ! q !(-1)^{p+q}} \\
& \times G_{2,3}^{3,1}\left(\frac{N_{T}}{\eta} \mid \begin{array}{c}
0,1 \\
0,0, p+q+\nu+1
\end{array}\right), \\
& \mathbb{E}\left[\mathcal{I}_{l}^{2}\right]=2 e^{\frac{N_{T}}{\eta}} \sum_{m=0}^{M-1} \sum_{p=0}^{m} \sum_{q=0}^{m} \sum_{j=0}^{p+q+\nu}\left[\frac{(-1)^{\nu-j} m !\left(\begin{array}{l}
m+\nu \\
m-p
\end{array}\right)}{\left(\frac{\eta}{N_{T}}\right)^{p+q+\nu+1}}\right. \\
& \left.\times \frac{\left(\begin{array}{c}
m+\nu \\
m-q
\end{array}\right)\left(\begin{array}{c}
p+q+\nu \\
j
\end{array}\right)}{(m+\nu) ! p ! q !} G_{3,4}^{4,0}\left(\frac{N_{T}}{\eta} \mid \begin{array}{c}
-j,-j,-j \\
0,-j-1,-j-1,-j-1
\end{array}\right)\right] \\
& -\sum_{j=0}^{M-1} \sum_{k=0}^{M-1} \frac{j ! k !}{(j+\nu) !(k+\nu) !}\left[\sum_{p=0}^{j} \sum_{q=0}^{k} \frac{\left(\begin{array}{l}
j+\nu \\
j-p
\end{array}\right)\left(\begin{array}{l}
k+\nu \\
k-q
\end{array}\right)}{p ! q !(-1)^{p+q}}\right. \\
& \left.\times G_{2,3}^{3,1}\left(\frac{N_{T}}{\eta} \mid \begin{array}{c}
0,1 \\
0,0, p+q+\nu+1
\end{array}\right)\right]^{2}+\left\{\mathbb{E}\left[\mathcal{I}_{l}\right]\right\}^{2},
\end{aligned}
$$

where $G$ is Meijer's $G$ function [23, pp. 1096, 9.301].

Proof: See Appendix D.

Lemma 5: The ACF of MIMO IMI, defined as $r_{\mathcal{I}}(i)=$ $\mathbb{E}\left[\mathcal{I}_{l} \mathcal{I}_{l-i}\right]$, is shown to be

$$
\begin{gathered}
r_{\mathcal{I}}(i)=\left\{\mathbb{E}\left[\mathcal{I}_{l}\right]\right\}^{2}+\sum_{j=M}^{\infty} \sum_{k=0}^{M-1}\left\{\frac{j ! k ! \varrho_{i}^{2(j-k)}}{(j+\nu) !(k+\nu) !} \times\right. \\
\left.\left[\sum_{p=0}^{j} \sum_{q=0}^{k} \frac{\left(\begin{array}{l}
j+\nu \\
j-p
\end{array}\right)\left(\begin{array}{l}
k+\nu \\
k-q
\end{array}\right)}{p ! q !(-1)^{p+q}} G_{2,3}^{3,1}\left(\frac{N_{T}}{\eta} \mid \begin{array}{c}
0,1 \\
0,0, p+q+\nu+1
\end{array}\right)\right]^{2}\right\} .
\end{gathered}
$$

Proof: By plugging (29) into (64), and using (76), we obtain (37) immediately.

With Lemmas 4 and 5, the NACF and the correlation coefficient can be calculated according to

$$
\tilde{r}_{\mathcal{I}}(i)=\frac{r_{\mathcal{I}}(i)}{\mathbb{E}\left[\mathcal{I}_{l}^{2}\right]}
$$

and

$$
\rho_{\mathcal{I}}(i)=\frac{r_{\mathcal{I}}(i)-\left\{\mathbb{E}\left[\mathcal{I}_{l}\right]\right\}^{2}}{\mathbb{E}\left[\mathcal{I}_{l}^{2}\right]-\left\{\mathbb{E}\left[\mathcal{I}_{l}\right]\right\}^{2}},
$$

by inserting (36) and (35) into (38), and (36), (35) and (37) into (39), respectively.

In general, it seems difficult to further simplify (36), (35) and (37). However, we note that

$$
\ln (1+\omega x) \approx \begin{cases}\omega x, & \omega \rightarrow 0 \\ \ln (\omega x), & \omega \rightarrow \infty\end{cases}
$$

Using (40), we obtain asymptotic closed-form expressions for the NACF, $\tilde{r}_{\mathcal{I}}(i)$, and the correlation coefficient, $\rho_{\mathcal{I}}(i)$, at low- and high-SNR regimes, as follows.

1) The Low-SNR Regime: If $\eta \rightarrow 0$, based on (40), (34) can be approximated by

$$
\mathcal{I}_{l} \approx \sum_{m=1}^{M} \frac{\eta}{N_{T}} \lambda_{m}(l)
$$

which is the same as the low-SNR approximation of $\mathcal{I}_{l}$ in a MIMO system with orthogonal space-time block code (OSTBC) transmission [29], due to $\sum_{m=1}^{M} \lambda_{m}(l)=\operatorname{tr}\left[\mathbf{H}_{l} \mathbf{H}_{l}^{\dagger}\right]=$ $\left\|\mathbf{H}_{l}\right\|_{F}^{2}$. Therefore, the NACF and correlation coefficient of interest are equal to those derived for the OSTBC-MIMO system at low SNRs, as stated in the following proposition.

Proposition 1: At the low-SNR regime, the NACF and the correlation coefficient are given by [29]

$$
\begin{aligned}
& \tilde{r}_{\mathcal{I}}(i) \approx \frac{N_{R} N_{T}+\varrho_{i}^{2}}{N_{R} N_{T}+1}, \\
& \rho_{\mathcal{I}}(i) \approx \varrho_{i}^{2} .
\end{aligned}
$$

2) The High-SNR Regime: If $\eta \rightarrow \infty$, based on (40), (34) can be approximated by

$$
\mathcal{I}_{l} \approx \sum_{m=1}^{M} \ln \left[\frac{\eta}{N_{T}} \lambda_{m}(l)\right]
$$

whose NACF and correlation coefficient are presented in the following theorem.

Theorem 3: At high SNRs, the NACF and the correlation coefficient are given by (45) and (46), respectively, where ${ }_{p} F_{q}\left(a_{1}, \cdots, a_{p} ; b_{1}, \cdots, b_{q} ; z\right)$ is the generalized hypergeometric function [23, pp. 1071, 9.14.1], $\zeta(\cdot, \cdot)$ is the Riemann zeta function, given by $\zeta(z, q)=\sum_{k=0}^{\infty} \frac{1}{(q+k)^{z}}[23$, pp. 1101, 9.521.1], and $\psi_{k}$ is the digamma function [23, pp. 954, 8.365.4].

Proof: See Appendix E.

Theorem 3 includes the high-SNR approximation for the OSTBC-MIMO system in [29] as a special case. In fact, with $M=1,(45)$ and (46) simplify to the corresponding resutls in [29] by replacing $N$ with $M N$, i.e.,

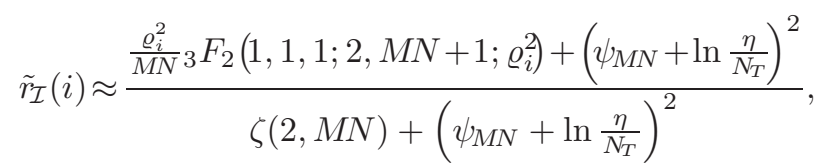

$\rho_{\mathcal{I}}(i) \approx \frac{\frac{\varrho_{i}^{2}}{M N}{ }_{3} F_{2}\left(1,1,1 ; 2, M N+1 ; \varrho_{i}^{2}\right)}{\zeta(2, M N)}$ 


$$
\begin{aligned}
& \tilde{r}_{\mathcal{I}}(i) \approx \frac{\sum_{m=0}^{M-1} \frac{M !(m+\nu) ! \varrho_{i}^{2(M-m)}{ }_{4} F_{3}\left(M-m, M-m, M+1,1 ; M-m+1, M-m+1, N+1 ; \varrho_{i}^{2}\right)}{(M-m)^{2} N ! m !}+\left(\sum_{m=0}^{M-1} \psi_{N-m}+M \ln \frac{\eta}{N_{T}}\right)^{2}}{\sum_{m=0}^{M-1} \zeta(2, N-m)+\left(\sum_{m=0}^{M-1} \psi_{N-m}+M \ln \frac{\eta}{N_{T}}\right)^{2}}, \\
& \rho_{\mathcal{I}}(i) \approx \frac{\sum_{m=0}^{M-1} \frac{M !(m+\nu) ! \varrho_{i}^{2(M-m)}}{(M-m)^{2} N ! m !}{ }_{4} F_{3}\left(M-m, M-m, M+1,1 ; M-m+1, M-m+1, N+1 ; \varrho_{i}^{2}\right)}{\sum_{m=0}^{M-1} \zeta(2, N-m)},
\end{aligned}
$$

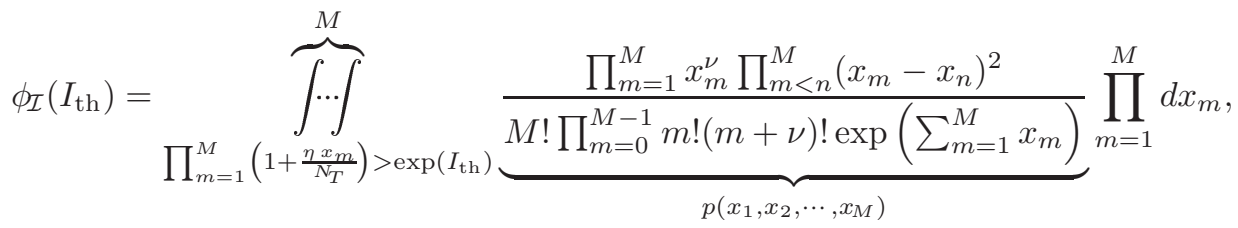

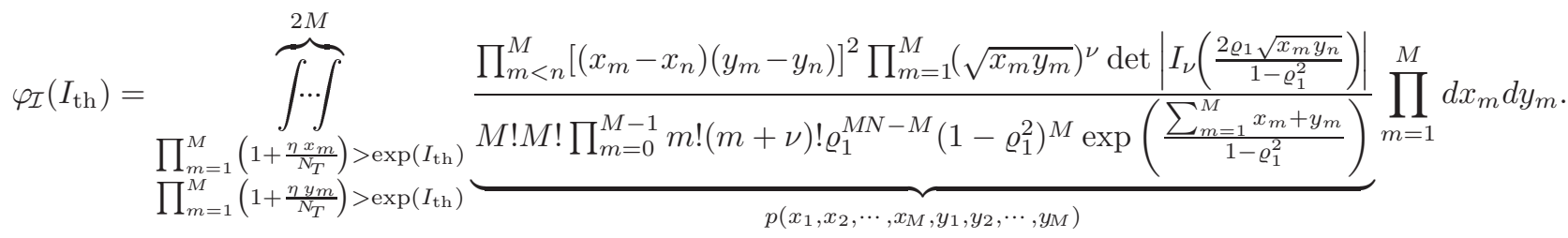

Table I

TAYLOR EXPANSION OF (46) AND THE MAXIMUM DifFERENCE BETWEEN (43) AND (46) FOR DiFFERENT $(M, N)$ 's

\begin{tabular}{|c||c|c|}
\hline$(M, N)$ & Taylor Series of $(46)$ & $\max _{0 \leq \varrho_{i} \leq 1}|(43)-(46)|^{\ddagger}$ \\
\hline \hline$(1,1)$ & $0.608 \varrho_{i}^{2}+0.152 \varrho_{i}^{4}+\mathcal{O}\left(\varrho_{i}^{6}\right)$ & 0.160 \\
\hline$(2,2)$ & $0.437 \varrho_{i}^{2}+0.218 \varrho_{i}^{4}+\mathcal{O}\left(\varrho_{i}^{6}\right)$ & 0.230 \\
\hline$(3,3)$ & $0.372 \varrho_{i}^{2}+0.186 \varrho_{i}^{4}+\mathcal{O}\left(\varrho_{i}^{6}\right)$ & 0.274 \\
\hline$(4,4)$ & $0.337 \varrho_{i}^{2}+0.168 \varrho_{i}^{4}+\mathcal{O}\left(\varrho_{i}^{6}\right)$ & 0.304 \\
\hline$(4,8)$ & $0.725 \varrho_{i}^{2}+0.178 \varrho_{i}^{4}+\mathcal{O}\left(\varrho_{i}^{6}\right)$ & 0.085 \\
\hline$\vdots$ & $\vdots$ & $\vdots$ \\
\hline$(4,12)$ & $0.824 \varrho_{i}^{2}+0.135 \varrho_{i}^{4}+\mathcal{O}\left(\varrho_{i}^{6}\right)$ & 0.050 \\
\hline$\vdots$ & $\vdots$ & $\vdots$ \\
\hline$(4,16)$ & $0.870 \varrho_{i}^{2}+0.107 \varrho_{i}^{4}+\mathcal{O}\left(\varrho_{i}^{6}\right)$ & 0.036 \\
\hline
\end{tabular}

$\ddagger$ The maximum difference is calculated via the function FindMaximum in Mathematica ${ }^{\circledR}$

where the identity ${ }_{4} F_{3}(1,1,1,2 ; 2,2, M N+1 ; x)=$ ${ }_{3} F_{2}(1,1,1 ; 2, M N+1 ; x)$ is used.

Based on Theorem 3, we conclude that if $\nu=0$ and $M \rightarrow$ $\infty,(46)$ reduces to

$$
\lim _{M \rightarrow \infty} \rho_{\mathcal{I}}(i)=\frac{-\ln \left(1-\varrho_{i}^{2}\right)}{\lim _{p \rightarrow \infty} \sum_{k=1}^{p} \frac{1}{k}}=\delta_{i, 0},
$$

where we the first "=" is obtained by collecting the terms in (46), and the second "=" is due to $\varrho_{i}<1, i \neq 0$. We conjecture that the second " $=$ " of (51) holds for any finite $\nu$ at high SNRs, i.e., $\lim _{M \rightarrow \infty} \rho_{\mathcal{I}}(i)=\delta_{i, 0}, \forall \nu<\infty$. It implies that MIMO IMI is asymptotically uncorrelated at high SNRs, if the difference between the numbers of $\mathrm{Tx}$ and $\mathrm{Rx}$ antennas is finite.

To better understand Theorem 3, the Taylor expansion of (46) and the maximum difference between (43) and (46) is listed in Table I, for different values of $M$ and $N$. From Table $\mathrm{I}$, the following observations can be made.
- If $\nu=N-M$ is fixed, the maximum difference between the low- and high-SNR approximations increases when $M$ increases, which is supported by the first four rows of Table I, i.e., $(M, N)=(1,1),(2,2),(3,3)$, and $(4,4)$.

- From the last several rows of Table I, i.e., $(M, N)=$ $(4,4),(4,8),(4,12)$ and $(4,16)$, one may conclude that if $M$ is fixed, the maximum difference between the lowand high-SNR approximations decreases as $\nu$ increases. Furthermore, $\rho_{\mathcal{I}}(i)$ can be well approximated by $\varrho_{i}^{2}$, with negligible error for any SNR, when $\frac{\nu}{M}$ is not small.

\section{B. LCR and AOD of MIMO IMI}

The technique developed in Subsection III-B is also valid for calculating the LCR and AOD of MIMO IMI, i.e., we can obtain them by replacing $\phi_{\lambda}\left(\lambda_{\mathrm{th}}\right)$ and $\varphi_{\lambda}\left(\lambda_{\mathrm{th}}\right)$ with $\phi_{\mathcal{I}}\left(I_{\mathrm{th}}\right)=$ $P_{r}\left\{\mathcal{I}_{l}>I_{\mathrm{th}}\right\}$ and $\varphi_{\mathcal{I}}\left(I_{\mathrm{th}}\right)=P_{r}\left\{\mathcal{I}_{l}>I_{\mathrm{th}}, \mathcal{I}_{l-1}>I_{\mathrm{th}}\right\}$ in $(25)$ and (31), respectively. Therefore, we only need to calculate, $\phi_{\mathcal{I}}\left(I_{\mathrm{th}}\right)$ and $\varphi_{\mathcal{I}}\left(I_{\mathrm{th}}\right)$, which are presented in the following theorem.

Theorem 4: At any given level $I_{\mathrm{th}}, \phi_{\mathcal{I}}\left(I_{\mathrm{th}}\right)$ and $\varphi_{\mathcal{I}}\left(I_{\mathrm{th}}\right)$ can be expressed in terms of multiple integrals, given by (47) and (48), respectively.

Proof: Let $\left\{X_{m}\right\}_{m=1}^{M}$ and $\left\{Y_{m}\right\}_{m=1}^{M}$ be $M$ unordered eigenvalues of $\mathbf{H}(l) \mathbf{H}^{\dagger}(l)$ and $\mathbf{H}(l-1) \mathbf{H}^{\dagger}(l-$ $1)$, respectively. Then the joint PDF of $\left\{X_{m}\right\}_{m=1}^{M}$ is given by $p\left(x_{1}, x_{2}, \cdots, x_{M}\right)$ in (47) [30], and the joint PDF of $\left\{X_{m}\right\}_{m=1}^{M}$ and $\left\{Y_{m}\right\}_{m=1}^{M}$ is given by $p\left(x_{1}, x_{2}, \cdots, x_{M}, y_{1}, y_{2}, \cdots, y_{M}\right)$ in (48) [22]. Moreover, according to (34), the event $\left\{\mathcal{I}_{l}>I_{\mathrm{th}}\right\}$ is equivalent to $\left\{\prod_{m=1}^{M}\left(1+\frac{\eta X_{m}}{N_{T}}\right)>e^{I_{\mathrm{th}}}\right\}$, which leads to (47). Similarly, one can see that the two events, $\left\{\mathcal{I}_{l}>I_{\mathrm{th}}, \mathcal{I}_{l-1}>I_{\mathrm{th}}\right\}$ and $\left\{\prod_{m=1}^{M}\left(1+\frac{\eta X_{m}}{N_{T}}\right)>e^{I_{\mathrm{th}}}, \prod_{m=1}^{M}\left(1+\frac{\eta Y_{m}}{N_{T}}\right)>e^{I_{\mathrm{th}}}\right\}$, have the same probability, which results in (48).

Although (47) and (48) can be used to calculate the LCR and AOD of MIMO IMI for small $M$ 's, e.g., $M=2$, via numerical 
multiple integrals, it is impractical for large $M$ 's. Fortunately, we can approximate $\mathcal{I}_{l}$ as a Gaussian random variable for large $M$ 's and $N$ 's, which is summarized in the following proposition.

Proposition 2: If $M$ and $N$ are large, $\mathcal{I}_{l}$ can be approximated as a Gaussian random variable with mean $\mu_{\mathcal{I}}=\mathbb{E}\left[\mathcal{I}_{l}\right]$ and variance $\sigma_{\mathcal{I}}^{2}=\mathbb{E}\left[\mathcal{I}_{l}^{2}\right]-\left\{\mathbb{E}\left[\mathcal{I}_{l}\right]\right\}^{2}$, where $\mathbb{E}\left[\mathcal{I}_{l}\right]$ and $\mathbb{E}\left[\mathcal{I}_{l}^{2}\right]$ are given by (35) and (36), respectively [16]-[18]. Moreover, we approximate $\mathcal{I}_{l}$ and $\mathcal{I}_{l-i}$ by a bivariate Gaussian random vector with mean $\left(\mathbb{E}\left[\mathcal{I}_{l}\right], \mathbb{E}\left[\mathcal{I}_{l}\right]\right)^{T}$ and the covariance matrix $\Sigma_{\mathcal{I}}=\sigma_{\mathcal{I}}^{2}\left(\begin{array}{cc}1 & \rho_{\mathcal{I}}(i) \\ \rho_{\mathcal{I}}(i) & 1\end{array}\right)$, where $\rho_{\mathcal{I}}(i)$ is presented in (39).

An intuition explanation for Proposition 2 is that according to (34), $\mathcal{I}_{l}$ is the sum of $M$ random variables. When $M$ is large, $\mathcal{I}_{l}$ becomes approximately Gaussian according to the central limit theorem. Based on Proposition 2, we have the following theorem for the LCR and AOD of MIMO IMI.

Theorem 5: Using the Gaussian approximation, we can express the LCR and AOD of MIMO IMI as

$$
\begin{aligned}
N_{\mathcal{I}}\left(I_{\mathrm{th}}\right) & =\frac{1}{\pi T_{s}} \int_{\frac{\pi}{4}+\frac{\arcsin \left[\rho_{\mathcal{I}}(1)\right]}{2}}^{\frac{\pi}{2}} \exp \left(-\frac{\tilde{I}_{\mathrm{th}}^{2}}{2 \sin ^{2} \theta}\right) d \theta, \\
\bar{t}_{\mathcal{I}}\left(I_{\mathrm{th}}\right) & =\frac{1-Q\left(\tilde{I}_{\mathrm{th}}\right)}{N_{\mathcal{I}}\left(I_{\mathrm{th}}\right)},
\end{aligned}
$$

where $\tilde{I}_{\mathrm{th}}=\frac{I_{\mathrm{th}}-\mu_{\mathcal{I}}}{\sigma_{\mathcal{I}}}$ is the normalized threshold, and $Q(x)=$ $\frac{1}{\sqrt{2 \pi}} \int_{x}^{\infty} e^{-\frac{t^{2}}{2}} d t$ is the Gaussian $Q$-function.

Proof: See Appendix F.

Theorem 5 requires $\mu_{\mathcal{I}}, \sigma_{\mathcal{I}}^{2}$ and $\rho_{\mathcal{I}}(1)$, which can be obtained from (35), (36) and (39). However, for low and high SNRs, we may use their corresponding approximations. For high SNRs, they are given by (83), (84) and (46), whereas for low SNRs we have $\mu_{\mathcal{I}}=\eta N_{R}, \sigma_{\mathcal{I}}^{2}=\frac{\eta^{2} N_{R}}{N_{T}}$ [29], and $\rho_{\mathcal{I}}(1)=\varrho_{1}^{2}$, obtained from (43). In practice, the LCR and AOD at $\mu_{\mathcal{I}}$, the ergodic capacity, are of interest, which simplify Theorem 5 considerably.

Corollary 1: The LCR and AOD of MIMO IMI at the level $\mu_{\mathcal{I}}$ are, respectively, given by

$$
\begin{aligned}
N_{\mathcal{I}}\left(\mu_{\mathcal{I}}\right) & =\frac{\pi-2 \arcsin \left[\rho_{\mathcal{I}}(1)\right]}{4 \pi T_{s}}, \\
\bar{t}_{\mathcal{I}}\left(\mu_{\mathcal{I}}\right) & =\frac{2 \pi T_{s}}{\pi-2 \arcsin \left[\rho_{\mathcal{I}}(1)\right]} .
\end{aligned}
$$

\section{Numerical Results AND Discussion}

In this paper, a generic power spectrum [19, (8)] [29] is used to simulate time-varying Rayleigh flat fading channels with nonisotropic scattering, according to the spectral method [31]. Similar to [29], to verify the accuracy of the derived formulas, we consider two types of scattering environments: isotropic scattering and nonisotropic scattering with three clusters of scatterers. For nonisotropic scattering, parameters of the three clusters are given by $\left[P_{1}, \kappa_{1}, \theta_{1}\right]=\left[\frac{1}{3}, 6,0\right]$, $\left[P_{2}, \kappa_{2}, \theta_{2}\right]=\left[\frac{1}{2}, 6, \frac{\pi}{4}\right]$, and $\left[P_{3}, \kappa_{3}, \theta_{3}\right]=\left[\frac{1}{6}, 8, \frac{25 \pi}{18}\right]$, respectively. In addition, in all the simulations, the maximum Doppler frequency $f_{D}$ is set to $10 \mathrm{~Hz}$, and $T_{s}=\frac{1}{20 f_{D}}$ seconds. The AoA distributions and the corresponding channel correlation coefficients for the above two scattering environments are plotted in Fig. 2.
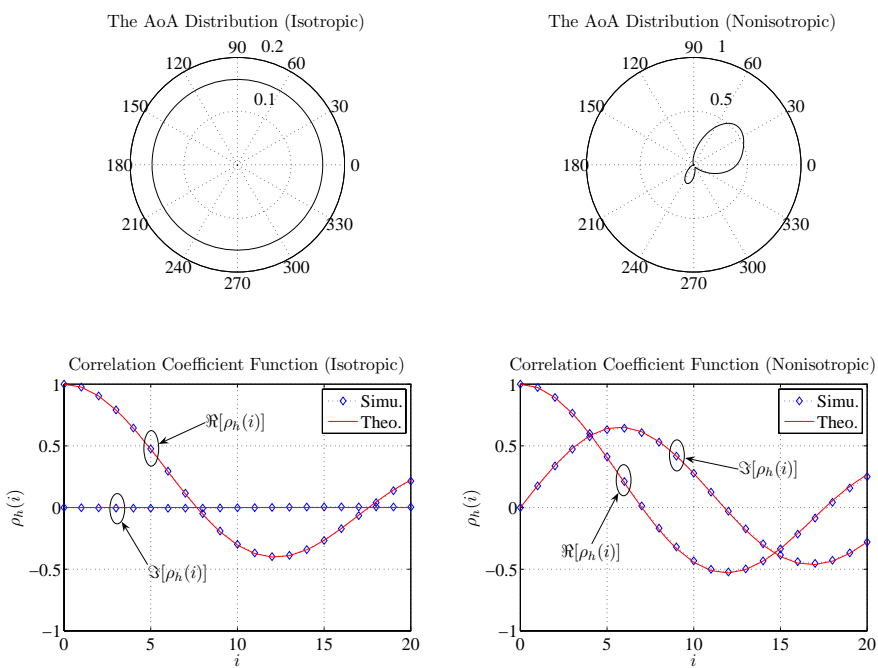

Figure 2. The AoA distributions for two scattering examples and the corresponding channel correlation coefficients.

In the following subsections, Monte Carlo simulations are performed to verify NACF, the correlation coefficient, LCR and AFD of eigen-channels and the MIMO IMI of two MIMO systems in the above two propagation environments: one is $4 \times 4$ and the other is $12 \times 3$. The NACF and the correlation coefficient bear almost the same information. The same comment applies to LCR and AFD. Therefore, we only report the simulation results for the correlation coefficient and the LCR, to save space.

\section{A. Eigen-Channels}

In this subsection, the correlation coefficient and the LCR of eigen-channels are considered for both isotropic and nonisotropic scattering environments.

1) Isotropic Scattering: This is Clarke's model [10], with uniform AoA. The comparison between the simulation and theoretical results is given in Fig. 3.
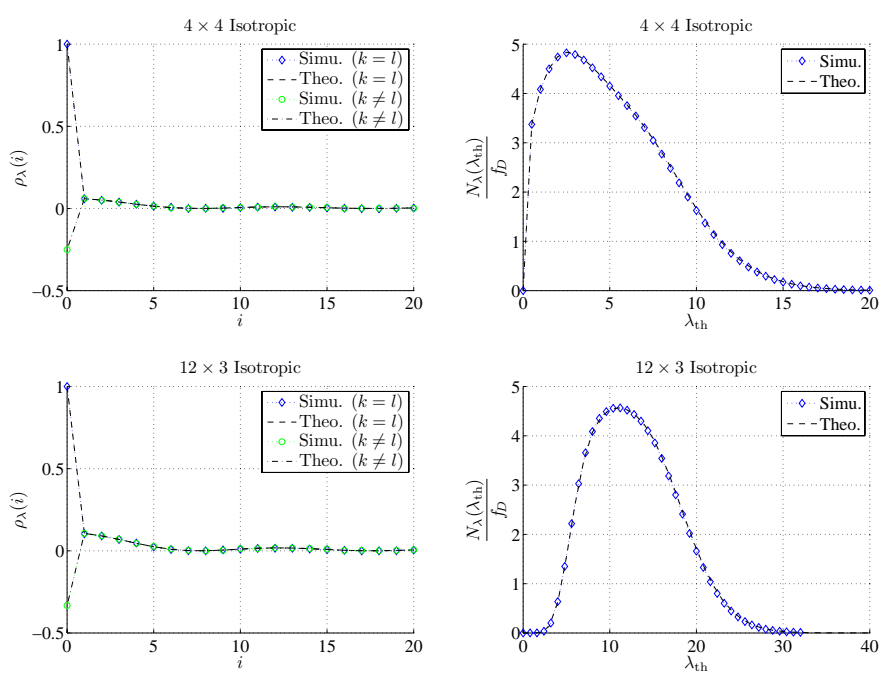

Figure 3. The correlation coefficient and the LCR of an eigen-channel, in $4 \times 4$ and $12 \times 3$ MIMO systems with isotropic scattering. 

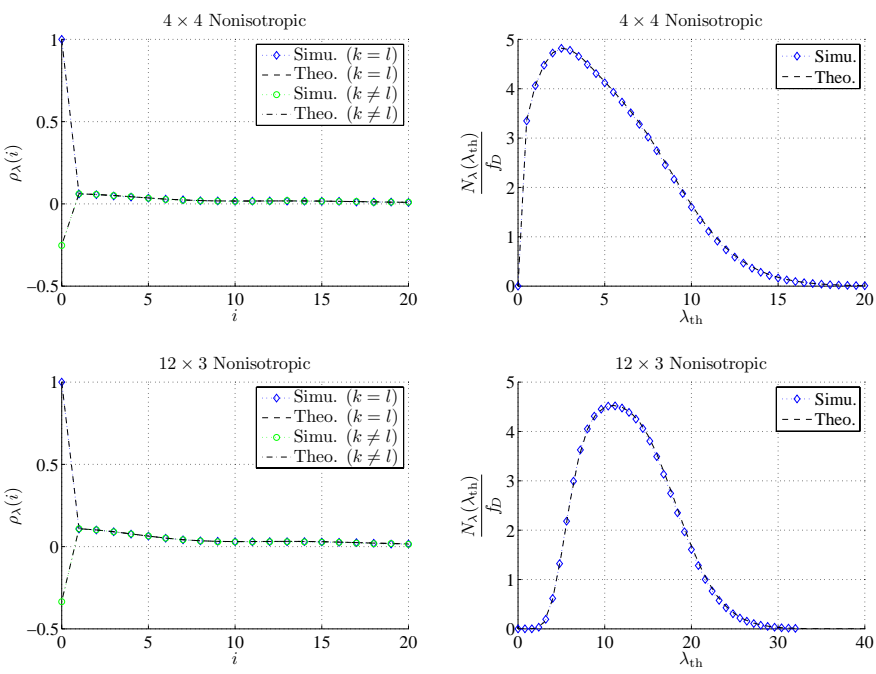

Figure 4. The correlation coefficient and the LCR of an eigen-channel, in $4 \times 4$ and $12 \times 3$ MIMO systems with nonisotropic scattering.

2) Nonisotropic Scattering: This is a general case, with an arbitrary AoA distribution [19][29]. The comparison results are presented in Fig. 4.

In Figs. 3 and 4, the upper left and right subfigures show the correlation coefficient and the LCR of eigen-channels in a $4 \times 4$ MIMO system, respectively, whereas the lower left and right subfigures show the results in a $12 \times 3$ MIMO system. In all figures, "Simu." means simulation. In the correlation coefficient plots, "Theo." means they are calculated according to (18), and " $(k=l)$ " denotes the autocorrelation coefficient, whereas " $(k \neq l)$ " indicates the cross-correlation coefficient. In the LCR plots, "Theo" indicates that the curve is computed using (25)-(28).

Based on the plots in Figs. 3 and 4, we can see that the derived analytical formulas perfectly match Monte Carlo simulations.

One possible application of correlation is power allocation in time-varying MIMO systems. Depending on the temporal correlation of eigen-channels, power allocation needs to be properly updated. For example, if temporal correlation is high, it means eigen-channels are not changing rapidly and therefore the chosen power allocation to different channels can be maintained for a certain period of time. On the other hand, if temporal correlation is low and eigen-channels are fluctuating fast, then one may need to change power allocation frequently.

\section{B. MIMO IMI}

In this subsection, the correlation coefficient and the LCR of MIMO IMI are presented for both isotropic and nonisotropic scattering environments at low- and high-SNR regimes. In the simulations and theoretical calculations, we set $\eta=-20 \mathrm{~dB}$ for low SNR, and $\eta=30 \mathrm{~dB}$ for high SNR.

1) Isotropic Scattering: For this case, the comparison results are shown in Fig. 5.

2) Nonisotropic Scattering: The comparison results regarding nonisotropic scattering are given in Fig. 6.

In Figs. 5 and 6, the upper three subfigures present the correlation coefficient and the LCR of the MIMO IMI in
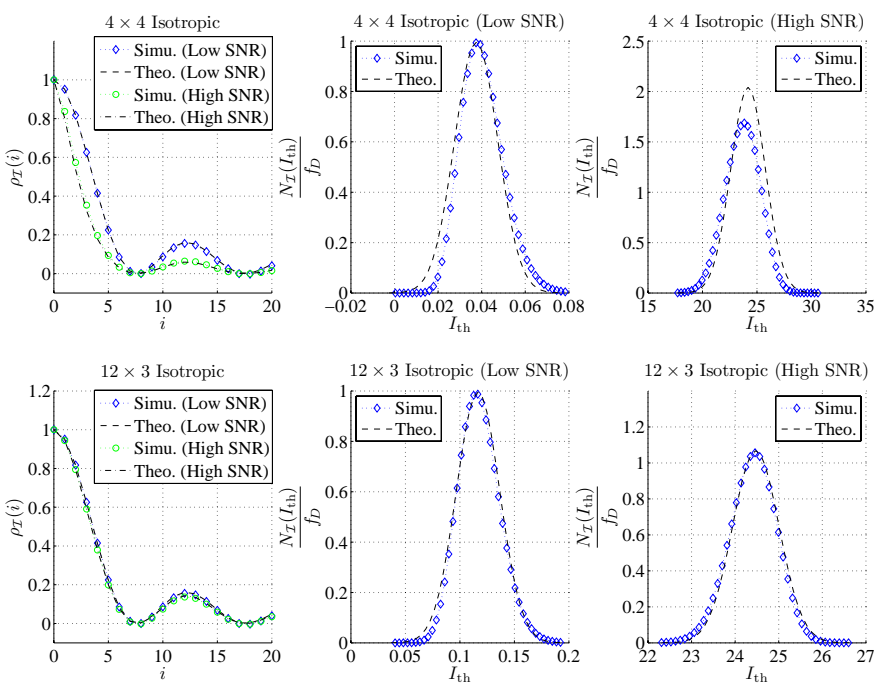

Figure 5. The correlation coefficient and the LCR of the MIMO IMI at lowand high-SNR regimes, in $4 \times 4$ and $12 \times 3$ MIMO systems with isotropic scattering.

a $4 \times 4$ system. Specifically, the upper left subfigure shows the correlation coefficient at low- and high-SNR regimes, the upper middle subfigure gives the LCR of the MIMO IMI at the low-SNR regime, whereas the upper right gives the LCR at the high-SNR regime. In addition, the lower three subfigures present the corresponding results in the $12 \times 3$ system. In the correlation coefficient plots, "Theo. (Low SNR)" corresponds to (43), whereas "Theo. (High SNR)" corresponds to (46). In the LCR plots, "Theo." means the values are computed from (52), where we used low- and high-SNR approximations for the mean $\mu_{\mathcal{I}}$ and variance $\sigma_{\mathcal{I}}^{2}$, listed immediately after Theorem 5.

From Figs. 5 and 6, the following observations can be made.

- Correlation coefficient: If $\nu=\max \left(N_{T}, N_{R}\right)-$ $\min \left(N_{T}, N_{R}\right)$ is large compared to $M=\min \left(N_{T}, N_{R}\right)$, we can approximate the correlation coefficient of MIMO
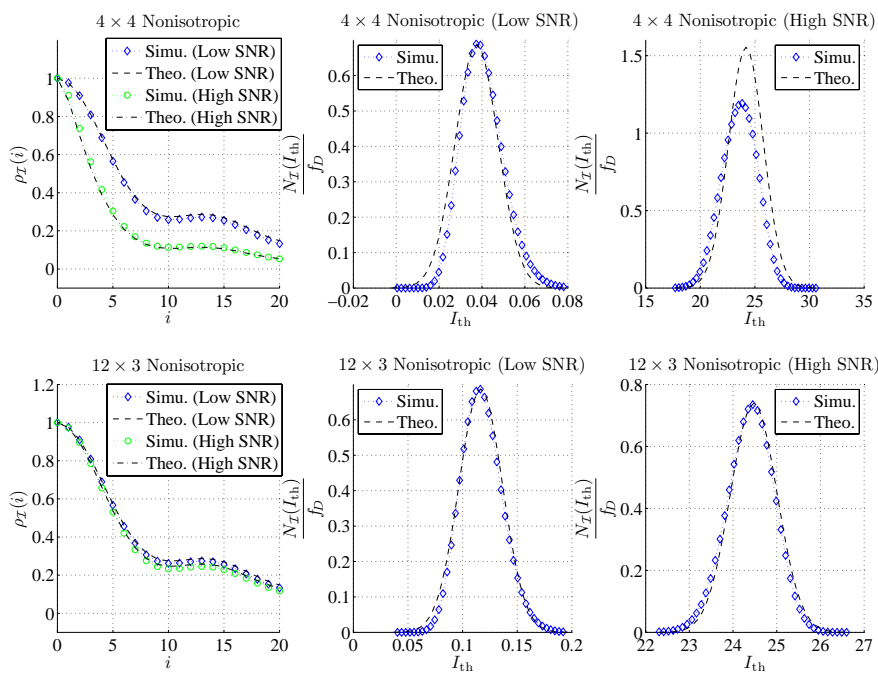

Figure 6. The correlation coefficient and the LCR of the MIMO IMI at lowand high-SNR regimes, in $4 \times 4$ and $12 \times 3$ MIMO systems with nonisotropic scattering. 


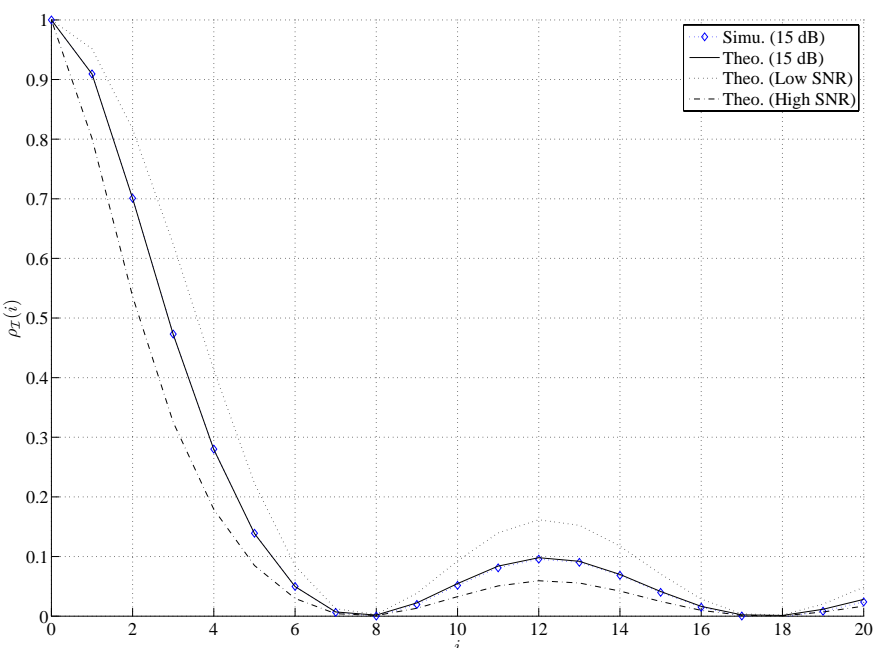

Figure 7. The correlation coefficient of the MIMO IMI at $\eta=15 \mathrm{~dB}$ (moderate SNR), in a $4 \times 4$ system with isotropic scattering.

IMI by the squared amplitude of the channel correlation coefficient for all SNRs, since low- and hign-SNR approximations are very close to each other (see the results for the $12 \times 3$ system). However, if $\nu$ is small compared to $M$, the gap between the low- and highSNR approximations is large (see the results for the $4 \times 4$ system). Therefore, we need to resort to the exact formulas in (36), (35), (37) and (39) to calculate the accurate values of the correlation coefficient, for not so small or large SNRs. For example, at $\eta=15 \mathrm{~dB}$, the simulation and exact theoretical curves, as well as lowand high-SNR approximations are shown in Fig. 7, for the correlation coefficient of the MIMO IMI in a $4 \times 4$ system.

- LCR: The Gaussian approximation works well at both low and high SNRs in large MIMO systems, e.g., the considered $12 \times 3$ channel. But it is not the case in small MIMO systems, say $4 \times 4$, where the Gaussian approximation has an obvious deviation from the simulation result at high SNR. This is because the central limit theorem does not hold for IMI in small MIMO systems ${ }^{4}$. For this case, we can numerically compute the multiple integrals given in (47) and (48), to calculate the LCR.

- LCR: Compared Fig. 3 and Fig. 4, we find the LCR of an eigen-channel is not sensitive to the scattering environment, which is not the case for the LCR of MIMO IMI. Furthermore, based on Figs. 5 and 6, we can see that the IMI in a nonisotropic scattering environment has less fluctuations than that in the isotropic scattering scenario.

The LCR of IMI can find applications in rate-adaptive systems. In such systems, IMI is fedback to the rate scheduler [16]. When IMI crosses a certain threshold, transmitted constellation needs to be changed accordingly [32]. The adaptation rate is given by the LCR of IMI. Using the derived formulas, a system designer can determine how adaptation rate may

\footnotetext{
${ }^{4}$ In fact, there are obvious differences between the true PDF and the Gaussian approximation in Fig. 1 of [17] at $\eta=15 \mathrm{~dB}$. Larger deviations are also observed at higher SNRs, say, $\eta=20 \mathrm{~dB}$, in Fig. 1 of [17].
}

depend on the physical parameters of the channel such as mean angle of arrivals and angle spreads. This can help the designer to optimize the design for a variety of propagation environments.

\section{CONCLUSION}

In this paper, closed-form expressions for several key second-order statistics such as the autocorrelation function, the correlation coefficient, level crossing rate and average fade/outage duration of eigen-channels and the instantaneous mutual information (IMI) are derived in MIMO time-varying Rayleigh flat fading channels.

Simulation and analytical results show that the eigenchannels tend to be spatio-temporally uncorrelated in large MIMO systems, and the correlation coefficient of the IMI can be well approximated by the squared amplitude of the correlation coefficient of the channel, if the difference between the number of $\mathrm{Tx}$ and $\mathrm{Rx}$ antennas is much larger than the minimum number of $\mathrm{Tx}$ and $\mathrm{Rx}$ antennas. In addition, we have also observed that the LCR of an eigen-channel is less sensitive to the scattering environment than the IMI.

The analytical expressions, supported by Monte Carlo simulations, provide quantitative information regarding the dynamic behavior of MIMO channels. They also serve as useful tools for MIMO system designs. For example, one may improve the performance of the feedbacked-IMI-based rate scheduler in a multiuser MIMO system by exploiting the temporal correlation of the IMI of each user.

\section{APPENDIX A \\ PROOF OF LEMMA 1}

Although the mean and second moment of $\lambda_{m}(l)$ were respectively given by (57) and (58) in [16] via a smart indirect method, we calculate them directly using its marginal PDF in (7), as follows.

Using 8.902.2 [23, pp. 1043], we can rewrite (7) as

$p(x)=\frac{(M-1) ! x^{\nu}}{(M+\nu-1) ! e^{x}}\left\{\left[L_{M-1}^{\nu}(x)\right]^{\prime} L_{M}^{\nu}(x)-\left[L_{M}^{\nu}(x)\right]^{\prime} L_{M-1}^{\nu}(x)\right\}$,

where' mean the derivative with respect to $x$. With [23, pp. $1062,8.971 .2]$

$$
\left[L_{n}^{\nu}(x)\right]^{\prime}=-L_{n-1}^{\nu+1}(x)
$$

and [23, pp. 1062, 8.971.5]

$$
L_{n}^{k}(x)=L_{n}^{k+1}(x)-L_{n-1}^{k+1}(x),
$$

(56) further reduces to

$$
p(x)=\frac{(M-1) ! x^{\nu}}{(M+\nu-1) ! e^{x}}\left\{\left[L_{M-1}^{\nu+1}(x)\right]^{2}-L_{M}^{\nu+1}(x) L_{M-2}^{\nu+1}(x)\right\},
$$

where the convention $L_{m}^{k}(x)=0, m<0$ should be used when it is applicable. 
Using (59), we obtain $\mathbb{E}\left[\lambda_{m}(l)\right]$ as

$$
\begin{aligned}
\mathbb{E}\left[\lambda_{m}(l)\right]= & \int_{0}^{\infty} x p(x) d x \\
= & \frac{(M-1) !}{(M+\nu-1) !}\left\{\int_{0}^{\infty} x^{\nu+1} e^{-x}\left[L_{M-1}^{\nu+1}(x)\right]^{2} d x\right. \\
& \left.-\int_{0}^{\infty} x^{\nu+1} e^{-x} L_{M}^{\nu+1}(x) L_{M-2}^{\nu+1}(x) d x\right\} \\
= & M+\nu,
\end{aligned}
$$

where the orthogonality of Laguerre polynomials [33, pp. 267, 7.414.3] is used, i.e.

$$
\int_{0}^{\infty} e^{-x} x^{\nu} L_{k}^{\nu}(x) L_{l}^{\nu}(x)=\frac{(k+\nu) !}{k !} \delta_{k, l} .
$$

The last line results in (13), considering $N=M+\nu$.

By substituting (58) with $k=\nu+1$ into (59) and using (61), we can easily obtain (14).

\section{APPENDIX B \\ PROOF OF LEMMA 2}

\section{A. The case of $i=0$}

For $i=0$, the value of $\mathbb{E}\left[\lambda_{m}^{2}(l)\right]$ is given in (14).

\section{B. The case of $i \neq 0$}

For $i \neq 0$, we need the following two lemmas.

Lemma 6: While $j, k$ and $\nu$ are non-negative integers, the value of the integral,

$I_{1}(j, k, \nu)=\int_{0}^{\infty} x^{\nu+1} e^{-x} L_{j}^{\nu}(x) L_{k}^{\nu}(x) d x$, is given by

$$
I_{1}(j, k, \nu)= \begin{cases}\frac{(2 k+\nu+1)(k+\nu) !}{k !}, & |j-k|=0, \\ -\frac{[\min (j, k)+\nu+1] !}{[\min (j, k)] !}, & |j-k|=1, \\ 0, & |j-k| \geq 2 .\end{cases}
$$

Proof: Using (58), we have

$$
\begin{aligned}
& L_{j}^{\nu}(x) L_{k}^{\nu}(x)=L_{j}^{\nu+1}(x) L_{k}^{\nu+1}(x)+L_{j-1}^{\nu+1}(x) L_{k-1}^{\nu+1}(x) \\
& \quad-L_{j}^{\nu+1}(x) L_{k-1}^{\nu+1}(x)-L_{j-1}^{\nu+1}(x) L_{k}^{\nu+1}(x) .
\end{aligned}
$$

Substitution of (63) into $I_{1}(j, k, \nu)$ results in (62), with the aid of (61) and the convention $L_{m}^{k}(x)=0, m<0$.

Lemma 7: The joint PDF in (6) can be written in the following equivalent form

$$
\begin{aligned}
p(x, y)=\frac{1}{M^{2}} & \sum_{j=M}^{\infty} \sum_{k=0}^{M-1}\left[\frac{j ! k ! \varrho_{i}^{2(j-k)}}{(j+\nu) !(k+\nu) !} \frac{x^{\nu} y^{\nu}}{e^{x+y}}\right. \\
& \left.\times L_{j}^{\nu}(x) L_{j}^{\nu}(y) L_{k}^{\nu}(x) L_{k}^{\nu}(y)\right]+p(x) p(y),
\end{aligned}
$$

where $p(\cdot)$ is the marginal PDF given by (7).

Proof: By applying the Hille-Hardy formula [33, pp. 185, (46)]

$$
\begin{aligned}
\sum_{k=0}^{\infty} \frac{k ! z^{k}}{(k+\nu) !} L_{k}^{\nu}(x) L_{k}^{\nu}(y)= & \frac{(x y z)^{-\frac{\nu}{2}}}{1-z} \exp \left(-z \frac{x+y}{1-z}\right) \\
& \times I_{\nu}\left(\frac{2 \sqrt{x y z}}{1-z}\right),|z|<1,
\end{aligned}
$$

to (6), we can obtain (64) through the following steps.

$$
\begin{aligned}
& \frac{(x y)^{\frac{\nu}{2}} e^{-\frac{x+y}{1-\varrho_{i}^{2}}} I_{\nu}\left(\frac{2 \varrho_{i} \sqrt{x y}}{1-\varrho_{i}^{2}}\right)}{M^{2}\left(1-\varrho_{i}^{2}\right) \varrho_{i}^{\nu}} \\
= & \frac{(x y)^{\nu}}{M^{2} e^{x+y}} \frac{\left(x y \varrho_{i}^{2}\right)^{-\frac{\nu}{2}} e^{-\varrho_{i}^{2} \frac{x+y}{1-\varrho_{i}^{2}}} I_{\nu}\left(\frac{2 \sqrt{x y \varrho_{i}^{2}}}{1-\varrho_{i}^{2}}\right)}{\left(1-\varrho_{i}^{2}\right)}, \\
= & \frac{x^{\nu} y^{\nu}}{M^{2} e^{x+y}} \sum_{j=0}^{\infty} \frac{j !}{(j+\nu) !} \varrho_{i}^{2 j} L_{j}^{\nu}(x) L_{j}^{\nu}(y), \\
= & \frac{x^{\nu} y^{\nu}}{M^{2} e^{x+y}}\left[\sum_{j=0}^{M-1} \frac{j !}{(j+\nu) !} \varrho_{i}^{2 j} L_{j}^{\nu}(x) L_{j}^{\nu}(y)\right. \\
& \left.+\sum_{j=M}^{\infty} \frac{j !}{(j+\nu) !} \varrho_{i}^{2 j} L_{j}^{\nu}(x) L_{j}^{\nu}(y)\right]
\end{aligned}
$$

Using (66), the first part of (6) can be written as

$$
\begin{aligned}
& \frac{(x y)^{\frac{\nu}{2}} e^{-\frac{x+y}{1-\varrho_{i}^{2}}} I_{\nu}\left(\frac{2 \varrho_{i} \sqrt{x y}}{1-\varrho_{i}^{2}}\right)}{M^{2}\left(1-\varrho_{i}^{2}\right) \varrho_{i}^{\nu}} \sum_{k=0}^{M-1} \frac{k ! L_{k}^{\nu}(x) L_{k}^{\nu}(y)}{(k+\nu) ! \varrho_{i}^{2 k}} \\
= & \frac{x^{\nu} y^{\nu}}{M^{2} e^{x+y}} \sum_{j=0}^{M-1} \frac{j ! \varrho_{i}^{2 j} L_{j}^{\nu}(x) L_{j}^{\nu}(y)}{(j+\nu) !} \sum_{k=0}^{M-1} \frac{k ! L_{k}^{\nu}(x) L_{k}^{\nu}(y)}{(k+\nu) ! \varrho_{i}^{2 k}} \\
& +\frac{x^{\nu} y^{\nu}}{M^{2} e^{x+y}} \sum_{j=M}^{\infty} \frac{j ! \varrho_{i}^{2 j} L_{j}^{\nu}(x) L_{j}^{\nu}(y)}{(j+\nu) !} \sum_{k=0}^{M-1} \frac{k ! L_{k}^{\nu}(x) L_{k}^{\nu}(y)}{(k+\nu) ! \varrho_{i}^{2 k}} \\
= & \frac{x^{\nu} y^{\nu}}{M^{2} e^{x+y}} \underbrace{M-1}_{j=0} \sum_{k=0}^{M-1} \frac{j ! k ! \varrho_{i}^{2(j-k)} L_{k}^{\nu}(x) L_{k}^{\nu}(y) L_{j}^{\nu}(x) L_{j}^{\nu}(y)}{(j+\nu) !(k+\nu) !} \\
& +\frac{x^{\nu} y^{\nu}}{M^{2} e^{x+y}} \sum_{j=M}^{\infty} \sum_{k=0}^{M-1} \frac{j ! k ! \varrho_{i}^{2(j-k)} L_{k}^{\nu}(x) L_{k}^{\nu}(y) L_{j}^{\nu}(x) L_{j}^{\nu}(y)}{(j+\nu) !(k+\nu) !}
\end{aligned}
$$

where the last part is the same as the first part of (64). In addition,

$$
\begin{aligned}
& \sum_{0 \leq k<l}^{M-1} \frac{k ! l !}{(k+\nu) !(l+\nu) !}\left\{\left[L_{k}^{\nu}(x) L_{l}^{\nu}(y)\right]^{2}+\left[L_{l}^{\nu}(x) L_{k}^{\nu}(y)\right]^{2}\right\} \\
= & \sum_{k=0}^{M-1} \sum_{l=k+1}^{M-1} \frac{k ! l !\left[L_{k}^{\nu}(x) L_{l}^{\nu}(y)\right]^{2}}{(k+\nu) !(l+\nu) !} \\
& +\sum_{l=0}^{M-1} \sum_{k=0}^{l-1} \frac{k ! l !\left[L_{l}^{\nu}(x) L_{k}^{\nu}(y)\right]^{2}}{(k+\nu) !(l+\nu) !} \\
= & \sum_{k=0}^{M-1} \sum_{l=k+1}^{M-1} \frac{k ! l !\left[L_{k}^{\nu}(x) L_{l}^{\nu}(y)\right]^{2}}{(k+\nu) !(l+\nu) !} \\
& +\sum_{k=0}^{M-1} \sum_{l=0}^{k-1} \frac{k ! l !\left[L_{k}^{\nu}(x) L_{l}^{\nu}(y)\right]^{2}}{(k+\nu) !(l+\nu) !} \\
= & \sum_{k=0}^{M-1} \sum_{l=0, l \neq k}^{M-1} \frac{k ! l !\left[L_{k}^{\nu}(x) L_{l}^{\nu}(y)\right]^{2}}{(k+\nu) !(l+\nu) !} .
\end{aligned}
$$

Note that after the first " $="$ sign in (68), the double summation $\sum_{0 \leq k<l}^{M-1}$ is written as the sum of $\sum_{k=0}^{M-1} \sum_{l=k+1}^{M-1}$ and 
$\sum_{l=0}^{M-1} \sum_{k=0}^{l-1}$, whereas in the second double summation after the second "=" sign in (68), the order of $k$ and $l$ is changed due to the symmetry of the summand with respect to $k$ and $l$. Similarly, one can have

$$
\begin{aligned}
& \sum_{0 \leq k<l}^{M-1} \frac{k ! l !\left[\varrho_{i}^{2(l-k)}+\varrho_{i}^{2(k-l)}\right] L_{k}^{\nu}(x) L_{l}^{\nu}(x) L_{k}^{\nu}(y) L_{l}^{\nu}(y)}{(k+\nu) !(l+\nu) !} \\
= & \underbrace{\sum_{k=0}^{M-1} \sum_{l=0, l \neq k}^{M-1} \frac{k ! l ! \varrho_{i}^{2(k-l)} L_{k}^{\nu}(x) L_{l}^{\nu}(x) L_{k}^{\nu}(y) L_{l}^{\nu}(y)}{(k+\nu) !(l+\nu) !}}_{\Theta(x, y)}
\end{aligned}
$$

Subtracting $\Theta(x, y)$ in (69) from $\Lambda(x, y)$ in (67) leads to $\Lambda(x, y)-\Theta(x, y)=\sum_{k=0}^{M-1} \sum_{l=k} \frac{\left.k ! l ! ! L_{k}^{\nu}(x) L_{l}^{\nu}(y)\right]^{2}}{(k+\nu) !(l+\nu) !}$. By adding this to (68) we obtain $\sum_{k=0}^{M-1} \frac{k !\left[L_{k}^{\nu}(x)\right]^{2}}{(k+\nu) !} \sum_{l=0}^{M-1} \frac{l !\left[L_{l}^{\nu}(y)\right]^{2}}{(l+\nu) !}$. Upon multiplying this by $\frac{x^{\nu} y^{\nu}}{M^{2} e^{x+y}}$, we obtain the second part of (64), which completes the proof.

Lemma 7 shows the joint PDF is the summation of a cross term and the product of two marginal PDFs. Therefore,

$$
\begin{aligned}
r_{m, m}(i)= & \mathbb{E}\left[\lambda_{m}(l) \lambda_{m}(l-i)\right] \\
= & \left\{\mathbb{E}\left[\lambda_{m}(l)\right]\right\}^{2}+\iint_{0}^{\infty} \frac{x y}{M^{2}} \sum_{j=M}^{\infty} \sum_{k=0}^{M-1}\left[\frac{j ! k ! \varrho_{i}^{2(j-k)}}{(j+\nu) !(k+\nu) !}\right. \\
& \left.\times \frac{x^{\nu} y^{\nu} L_{j}^{\nu}(x) L_{j}^{\nu}(y) L_{k}^{\nu}(x) L_{k}^{\nu}(y)}{e^{x+y}}\right] d x d y, \\
= & N^{2}+\sum_{j=M}^{\infty} \sum_{k=0}^{M-1}\left\{\frac{j ! k ! \varrho_{i}^{2(j-k)}}{M^{2}(j+\nu) !(k+\nu) !}\right. \\
& \left.\times\left[\int_{0}^{\infty} x^{\nu+1} e^{-x} L_{j}^{\nu}(x) L_{k}^{\nu}(x) d x\right]^{2}\right\} \\
= & N^{2}+\sum_{j=M}^{\infty} \sum_{k=0}^{M-1} \frac{j ! k ! \varrho_{i}^{2(j-k)}\left[I_{1}(j, k, \nu)\right]^{2}}{M^{2}(j+\nu) !(k+\nu) !} \\
= & N^{2}+\frac{1}{M^{2}} \frac{M !(M-1) ! \varrho_{i}^{2}\left[I_{1}(M, M-1, \nu)\right]^{2}}{(M+\nu) !(M+\nu-1) !},
\end{aligned}
$$

where the last result is based on $I_{1}(j, k, \nu)=0, \forall|j-k| \geq 2$, according to Lemma 6. It further reduces to (15), based on (62) and $N=M+\nu$.

\section{APPENDix C \\ PROOF OF LEMMA 3}

\section{A. The case of $i=0$}

For $i=0$, we need the following proposition.

Proposition 3: If $\left(x_{1}, x_{2}\right)$ are a pair of eigenvalues of the set $\left\{\lambda_{m}(l)\right\}_{m=1}^{M}$, then their joint PDF is given by [22]

$$
\begin{aligned}
& p\left(x_{1}, x_{2}\right)=\frac{\left(x_{1} x_{2}\right)^{\nu} e^{-\left(x_{1}+x_{2}\right)}}{M(M-1)} \sum_{\substack{p, q=0 \\
p \neq q}}^{M-1} \frac{p ! q !}{(p+\nu) !(q+\nu) !} \\
& \times\left\{\left[L_{p}^{\nu}\left(x_{1}\right) L_{q}^{\nu}\left(x_{2}\right)\right]^{2}-L_{p}^{\nu}\left(x_{1}\right) L_{q}^{\nu}\left(x_{1}\right) L_{p}^{\nu}\left(x_{2}\right) L_{q}^{\nu}\left(x_{2}\right)\right\} .
\end{aligned}
$$

Note that (71) is different from (6). By reordering the items, we can rewrite (71) as

$$
\begin{gathered}
p\left(x_{1}, x_{2}\right)=\frac{M}{M-1} p\left(x_{1}\right) p\left(x_{2}\right)-\frac{\left(x_{1} x_{2}\right)^{\nu} e^{-\left(x_{1}+x_{2}\right)}}{M(M-1)} \\
\times \sum_{j=0}^{M-1} \sum_{k=0}^{M-1} \frac{j ! k ! L_{j}^{\nu}\left(x_{1}\right) L_{k}^{\nu}\left(x_{1}\right) L_{j}^{\nu}\left(x_{2}\right) L_{k}^{\nu}\left(x_{2}\right)}{(j+\nu) !(k+\nu) !} .
\end{gathered}
$$

Using Lemma 6 and (72), it is easy to obtain

$$
r_{m, n}(i)=\frac{M}{M-1} N^{2}-\frac{S}{M(M-1)},
$$

where $S=\sum_{j=0}^{M-1} \sum_{k=0}^{M-1} \frac{j ! k !\left[I_{1}(j, k, \nu)\right]^{2}}{(j+\nu) !(k+\nu) !}$. According to (62), we have

$$
\begin{aligned}
S & =\sum_{k=0}^{M-1}(2 k+\nu+1)^{2}+2 \sum_{k=0}^{M-2}(k+1)(k+\nu+1), \\
& =M N(M+N-1),
\end{aligned}
$$

where the last line is derived based on $\sum_{k=0}^{n} k=\frac{n(n+1)}{2}$ [23, pp. 2, 0.121.1] and $\sum_{k=0}^{n} k^{2}=\frac{n(n+1)(2 n+1)}{6}$ [23, pp. 2, $0.121 .2]$.

Substitution of (74) into (73) proves the first part of Lemma 3 , i.e., $i=0$. Note that the same result was derived in Lemma $A$ of [16] via an indirect method.

\section{B. The case of $i \neq 0$}

Note that $\left\{\lambda_{m}(l)\right\}_{m=1}^{M}$ and $\left\{\lambda_{m}(l-i)\right\}_{m=1}^{M}$ are unordered eigenvalues of $\mathbf{H}(l) \mathbf{H}^{\dagger}(l)$ and $\mathbf{H}(l-i) \mathbf{H}^{\dagger}(l-i)$, respectively, for $i \neq 0$. So the bivariate PDF of $\left\{\lambda_{m}(l), \lambda_{n}(l-i)\right\}, m \neq n$, is the same as that of $\left\{\lambda_{m}(l), \lambda_{m}(l-i)\right\}$, the latter given in (6). Therefore, $r_{m, n}(i)=r_{m, m}(i), i \neq 0, \forall m, n$, where $r_{m, m}(i)=N^{2}+\frac{N \varrho_{i}^{2}}{M}, i \neq 0$, is proved in Appendix B.

\section{APPENDIX D \\ PROOF OF LEMMA 4}

According to (34), we have

$$
\begin{aligned}
\mathbb{E}\left[\mathcal{I}_{l}\right] & =M \mathbb{E}\left[\ln \left(1+\frac{\eta}{N_{T}} \lambda_{m}(l)\right)\right], \\
& =M \int_{0}^{\infty} \ln \left(1+\frac{\eta}{N_{T}} x\right) p(x) d x,
\end{aligned}
$$

where $p(x)$ is given in (7). Substitution of (7) and (29) into (75) results in (35), with the aid of the following integral identity [29]

$$
\int_{0}^{\infty} x^{k} e^{-x} \ln (1+\omega x) \mathrm{d} x=G_{2,3}^{3,1}\left(\frac{1}{\omega} \mid \begin{array}{c}
0,1 \\
0,0, k+1
\end{array}\right),
$$

where $G$ is Meijer's $G$ function [23, pp. 1096, 9.301]. Eq. (76) is derived using $e^{-x}=G_{0,1}^{1,0}\left(\begin{array}{l}x \\ 0\end{array}\right), \ln (1+\omega x)=$ $G_{2,2}^{1,2}\left(\omega x \mid \begin{array}{l}1,1 \\ 1,0\end{array}\right)$ [34, (11)], the integral in [34, (21)], and $z^{k} G_{p, q}^{m, n}\left(z \mid \begin{array}{c}\left(a_{p}\right) \\ \left(b_{q}\right)\end{array}\right)=G_{p, q}^{m, n}\left(z \mid \begin{array}{l}k+\left(a_{p}\right) \\ k+\left(b_{q}\right)\end{array}\right)$ [35, pp. 521, 8.2.2.15], in which $\left(a_{p}\right)=a_{1}, a_{2}, \cdots, a_{p}$ and $\left(b_{q}\right)=$ $b_{1}, b_{2}, \cdots, b_{q}$. 
Similarly, we have

$$
\begin{aligned}
\mathbb{E}\left[\mathcal{I}_{l}^{2}\right]= & M \mathbb{E}\left[\ln ^{2}\left(1+\frac{\eta}{N_{T}} \lambda_{m}(l)\right)\right]+M(M-1) \\
& \times \underbrace{\mathbb{E}\left[\ln \left(1+\frac{\eta}{N_{T}} \lambda_{m}(l)\right) \ln \left(1+\frac{\eta}{N_{T}} \lambda_{n}(l)\right)\right]}_{m \neq n}, \\
= & M \int_{0}^{\infty} \ln ^{2}\left(1+\frac{\eta}{N_{T}} x\right) p(x) d x+M(M-1) \\
& \times \iint_{0}^{\infty} \ln \left(1+\frac{\eta}{N_{T}} x_{1}\right) \ln \left(1+\frac{\eta}{N_{T}} x_{2}\right) p\left(x_{1}, x_{2}\right) d x_{1} d x_{2},
\end{aligned}
$$

where $p(x)$ and $p\left(x_{1}, x_{2}\right)$ are given in (7) and (72), respectively. Substitution of (7), (29) and (72) into (77) leads us to (36), upon using (76) and the following integral equality [29]

$$
\begin{aligned}
& \int_{0}^{\infty} x^{k} e^{-x} \ln ^{2}(1+\omega x) \mathrm{d} x \\
& z=1+\omega x \frac{e^{\frac{1}{\omega}} \sum_{j=0}^{k}\left(\begin{array}{c}
k \\
j
\end{array}\right)(-1)^{k-j} \int_{1}^{\infty} z^{j} e^{-\frac{z}{\omega}} \ln ^{2} z \mathrm{~d} z}{\omega^{k+1}}, \\
& =\frac{\left.e^{\frac{1}{\omega}} \sum_{j=0}^{k}\left(\begin{array}{c}
k \\
j
\end{array}\right)(-1)^{k-j} \frac{\partial^{2}}{\partial \nu^{2}}\left[\omega^{\nu} \Gamma\left(\nu, \frac{1}{\omega}\right)\right]\right|_{\nu=j+1}}{\omega^{k+1}}, \\
& =\frac{2 e^{\frac{1}{\omega}} \sum_{j=0}^{k}\left(\begin{array}{c}
k \\
j
\end{array}\right)(-1)^{k-j} G_{3,4}^{4,0}\left(\frac{1}{\omega} \mid \begin{array}{c}
-j,-j,-j \\
0,-j-1,-j-1,-j-1
\end{array}\right)}{\omega^{k+1}},
\end{aligned}
$$

where in the first result the binomial expansion is used: $(x+$ $a)^{k}=\sum_{j=0}^{k}\left(\begin{array}{c}k \\ j\end{array}\right) x^{j}(-1)^{k-j}$ [23, pp. 26], the second result is obtained based on the integral identity 4.358.1 in [23, pp. 607], and the last result is derived with the aid of Eqs. (29) and (39) in [36] and $z^{k} G_{p, q}^{m, n}\left(z \mid \begin{array}{c}\left(a_{p}\right) \\ \left(b_{q}\right)\end{array}\right)=G_{p, q}^{m, n}\left(z \mid \begin{array}{c}k+\left(a_{p}\right) \\ k+\left(b_{q}\right)\end{array}\right)$ [35, pp. 521, 8.2.2.15].

\section{APPENDIX E \\ PROOF OF THEOREM 3}

First we derive the expressions for the first and second moments of $\mathcal{I}_{l}$ in (44), based on the following lemma.

Lemma 8: Let $\mathbf{X}=\left(x_{m, n}\right)$ be a random matrix with $M$ rows and $N$ columns, $M \leq N$, where each element is a zero mean unit variance complex Gaussian random variable and all the $N$ columns are i.i.d $M$-variate random vectors with the same $M \times M$ positive definite covariance matrix $\Sigma$. The mean and variance of $\ln \operatorname{det}\left(\mathbf{X X}^{\dagger}\right)$ are

$$
\begin{aligned}
\mathbb{E}\left[\ln \operatorname{det}\left(\mathbf{X X}^{\dagger}\right)\right] & =\sum_{m=0}^{M-1} \psi_{N-m}+\ln \operatorname{det} \Sigma, \\
\operatorname{var}\left[\ln \operatorname{det}\left(\mathbf{X X}^{\dagger}\right)\right] & =\sum_{m=0}^{M-1} \zeta(2, N-m) .
\end{aligned}
$$

Proof: According to Theorem 1.1 of [37], $\frac{\operatorname{det}\left(\mathbf{X X}^{\dagger}\right)}{2^{-M} \operatorname{det} \Sigma}$ has the same distribution as the product of $M$ independent $\chi^{2}$ random variables with $2 N, 2(N-1), \cdots, 2(N-M+1)$ degrees of freedom, respectively. Therefore, we can express $\ln \operatorname{det}\left(\mathbf{X X}^{\dagger}\right)$ as

$$
\ln \operatorname{det}\left(\mathbf{X X}^{\dagger}\right) \stackrel{\mathrm{d}}{=} \sum_{m=0}^{M-1} \ln \left(\sqrt[M]{\operatorname{det} \Sigma} y_{m}\right)
$$

where the notation $\stackrel{\mathrm{d}}{=}$ indicates "equal to in distribution", $2 y_{m}$ is a $\chi^{2}$ random variable with $2(N-m)$ degrees of freedom, and $\left\{y_{m}\right\}_{m=0}^{M-1}$ are independent.

Based on the results in [29], we have $\mathbb{E}\left[\ln y_{m}\right]=$ $H_{N-m-1}-\mathrm{C}$ and $\operatorname{var}\left[\ln y_{m}\right]=\zeta(2, N-m)$, where $H_{k}$ is the $k^{\text {th }}$ harmonic number [38, pp. 29, (2.13)], defined by $H_{k}=\sum_{j=1}^{k} \frac{1}{j}$ for $k \geq 1$ with $H_{0}=0$, and $\mathrm{C}=0.577215 \cdots$ is the Euler-Mascheroni constant [23, pp. xxx]. This completes the proof if we note $\psi_{k+1}=H_{k}-\mathrm{C}$ [23, pp. 952, 8.365.4].

It is interesting to observe that the correlation matrix $\Sigma$ affects the mean of ln $\operatorname{det}\left(\mathbf{X X}^{\dagger}\right)$ in (79), but has no impact on its variance in (80).

According to Theorem 1.1.2 of [39] we have

$$
\prod_{m=1}^{M} \lambda_{m}(l)=\left\{\begin{array}{l}
\operatorname{det}\left(\mathbf{H}_{l} \mathbf{H}_{l}^{\dagger}\right), N_{R} \leq N_{T}, \\
\operatorname{det}\left(\mathbf{H}_{l}^{\dagger} \mathbf{H}_{l}\right), N_{R}>N_{T} .
\end{array}\right.
$$

By applying Lemma 8 and (82) to (44) with $\Sigma=\mathbf{I}_{M}$, we have the mean of $\mathcal{I}_{l}$ given below

$$
\begin{aligned}
\mathbb{E}\left[\mathcal{I}_{l}\right] & \approx \mathbb{E}\left\{\sum_{m=1}^{M} \ln \left[\frac{\eta}{N_{T}} \lambda_{m}(l)\right]\right\}, \\
& =\mathbb{E}\left[\ln \prod_{m=1}^{M} \lambda_{m}(l)\right]+M \ln \frac{\eta}{N_{T}}, \\
& \stackrel{(82)}{=} \mathbb{E}\left[\ln \operatorname{det}\left(\mathbf{H}_{l}^{\dagger} \mathbf{H}_{l}\right)\right]+M \ln \frac{\eta}{N_{T}}, \\
& \stackrel{\text { Lemma }}{\Sigma=} 8 \sum_{m=0}^{M-1} \psi_{N-m}+M \ln \frac{\eta}{N_{T}},
\end{aligned}
$$

where the third result assumes $N_{R}>N_{T}$, without loss of generality. Similarly, the variance of $\mathcal{I}_{l}$ is given by

$$
\begin{aligned}
\operatorname{var}\left[\mathcal{I}_{l}\right] & \approx \operatorname{var}\left[M \ln \frac{\eta}{N_{T}}+\ln \operatorname{det}\left(\mathbf{H}_{l}^{\dagger} \mathbf{H}_{l}\right)\right], \\
& =\operatorname{var}\left[\ln \operatorname{det}\left(\mathbf{H}_{l}^{\dagger} \mathbf{H}_{l}\right)\right], \\
& =\sum_{m=0}^{M-1} \zeta(2, N-m),
\end{aligned}
$$

where the constant term $M \ln \frac{\eta}{N_{T}}$ does not affect the variance. These two are consistent with the results in [16], where an implicit complex extension of Theorem 3.3.4 of [39] was used. Clearly, the second moment of $\mathcal{I}_{l}$ is given by

$$
\begin{aligned}
\mathbb{E}\left[\mathcal{I}_{l}^{2}\right] & =\left\{\mathbb{E}\left[\mathcal{I}_{l}\right]\right\}^{2}+\operatorname{var}\left[\mathcal{I}_{l}\right] \\
& \approx \sum_{m=0}^{M-1} \zeta(2, N-m)+\left(\sum_{m=0}^{M-1} \psi_{N-m}+M \ln \frac{\eta}{N_{T}}\right)^{2} .
\end{aligned}
$$

For calculating the autocorrelation of $\mathcal{I}_{l}$, we need the following lemma. 
Lemma 9: With $j, k$ and $\nu$ as non-negative integers and $j \neq k$, the value of the integral

$I_{2}(j, k, \nu)=\int_{0}^{\infty}(\ln x) x^{\nu} e^{-x} L_{j}^{\nu}(x) L_{k}^{\nu}(x) d x$ is given by

$$
I_{2}(j, k, \nu)=\frac{[\min (j, k)+\nu] !}{[\min (j, k)] ![\min (j, k)-\max (j, k)]} .
$$

Proof: First we consider $j>k$. Substitution of $L_{k}^{\nu}(x)$ with (29) into $I_{2}(j, k, \nu)$ gives

$$
\begin{aligned}
I_{2}(j, k, \nu) & =\sum_{p=0}^{k}\left(\begin{array}{l}
k+\nu \\
k-p
\end{array}\right) \frac{(-1)^{p}}{p !} \int_{0}^{\infty}(\ln x) x^{p+\nu} e^{-x} L_{j}^{\nu}(x) d x \\
& =\sum_{p=0}^{k}\left(\begin{array}{l}
k+\nu \\
k-p
\end{array}\right) \frac{(-1)^{p}(-1)^{p-1} p !(j-p-1) !(p+\nu) !}{p !} \\
& =-\frac{(k+\nu) !}{j !} \sum_{p=0}^{k} \frac{(j-p-1) !}{(k-p) !}
\end{aligned}
$$

where the second line comes from 2.19.6.2 [40, pp. 469]. Using $\sum_{q=0}^{m}\left(\begin{array}{c}n+q \\ n\end{array}\right)=\left(\begin{array}{c}n+m+1 \\ n+1\end{array}\right)$ [23, pp. 4, 0.151.1], we have $\sum_{p=0}^{k} \frac{(j-p-1) !}{(k-p) !} \stackrel{q=k-p}{=} \sum_{q=0}^{k}\left(\begin{array}{c}j-k-1-q \\ j-k-1\end{array}\right)(j-k-1) !=\left(\begin{array}{c}j \\ j-k\end{array}\right)(j-$ $k-1) !=\frac{j !}{k !(j-k)}$, which reduces $(87)$ to

$$
I_{2}(j, k, \nu)=\frac{(k+\nu) !}{k !(k-j)} .
$$

Similarly, for $j<k$, we obtain

$$
I_{2}(j, k, \nu)=\frac{(j+\nu) !}{j !(j-k)} .
$$

Combination of (88) and (89) results in (86).

Now we proceed to prove (45) and (46). Based on the highSNR approximation of $\mathcal{I}_{l}$ in (44), we have

$$
\begin{aligned}
r_{\mathcal{I}}(i) & \approx \sum_{m=1}^{M} \sum_{n=1}^{M} \mathbb{E}\left[\ln \frac{\eta \lambda_{m}(l)}{N_{T}} \ln \frac{\eta \lambda_{n}(l-i)}{N_{T}}\right], \\
& =M^{2} \mathbb{E}\left[\ln \frac{\eta \lambda_{1}(l)}{N_{T}} \ln \frac{\eta \lambda_{1}(l-i)}{N_{T}}\right], \\
& =M^{2}\left[\ln ^{2} \frac{\eta}{N_{T}}+2 \ln \frac{\eta}{N_{T}} \mathbb{E}[\ln \lambda(l)]+r_{\ln \lambda}(i)\right],
\end{aligned}
$$

where $r_{\ln \lambda}(i)=\mathbb{E}[\ln \lambda(l) \ln \lambda(l-i)]$. Using (64) and Lemma 9, $r_{\ln \lambda}(i)$ can be evaluated as

$$
r_{\ln \lambda}(i)=\sum_{j=M}^{\infty} \sum_{k=0}^{M-1} \frac{j ! k ! \varrho_{i}^{2(j-k)} I_{2}^{2}(j, k, \nu)}{M^{2}(j+\nu) !(k+\nu) !}+\{\mathbb{E}[\ln \lambda(l)]\}^{2} .
$$

By substituting (86) and (91) into (90), we obtain

$$
r_{\mathcal{I}}(i) \approx \sum_{k=0}^{M-1} S\left(k, \nu, \varrho_{i}\right)+\left\{\mathbb{E}\left[\mathcal{I}_{l}\right]\right\}^{2}
$$

where $S\left(k, \nu, \varrho_{i}\right)=\sum_{j=M}^{\infty} \frac{j !(k+\nu) ! \varrho_{i}^{2(j-k)}}{k !(j+\nu) !(j-k)^{2}}$, and $\mathbb{E}\left[\mathcal{I}_{l}\right] \approx$ $M \mathbb{E}\left[\ln \frac{\eta \lambda(l)}{N_{T}}\right]$ is approximated by (83). By introducing a new variable $p=j-M$ in $S\left(k, \nu, \varrho_{i}\right)$ and using the Pochhammer symbol $(x)_{n}=x(x+1) \cdots(x+n-1)$, we can rewrite $S\left(k, \nu, \varrho_{i}\right)$ as

$$
\begin{aligned}
S\left(k, \nu, \varrho_{i}\right)= & \frac{M !(k+\nu) ! \varrho_{i}^{2(M-k)}}{(M-k)^{2} N ! k !} \\
& \times \sum_{p=0}^{\infty} \frac{\left[(M-k)_{p}\right]^{2}(M+1)_{p}(1)_{p}}{\left[(M-k+1)_{p}\right]^{2}(N+1)_{p}} \frac{\varrho_{i}^{2 p}}{p !} \\
= & \frac{M !(k+\nu) ! \varrho_{i}^{2(M-k)}}{(M-k)^{2} N ! k !}{ }_{4} F_{3}(M-k, M-k, \\
& \left.M+1,1 ; M-k+1, M-k+1, N+1 ; \varrho_{i}^{2}\right),
\end{aligned}
$$

where $N=M+\nu$, and the last line comes from the definition of the generalized hypergeometric function [23, pp. 1071, 9.14.1].

Substitution of (85), (92) and (93) into (38) results in (45). Similarly, with (83), (84), (92) and (93), (39) reduces to (46).

\section{APPENDIX F}

\section{PROOF OF THEOREM 5}

To simplify the notation, we set $X=\mathcal{I}_{l}, Y=\mathcal{I}_{l-1}$, and $\rho=\rho_{\mathcal{I}}(1)$. According to Proposition 2, we have the PDF of $X$ and the joint PDF of $X$ and $Y$ as

$$
p(x)=\frac{1}{\sqrt{2 \pi} \sigma_{\mathcal{I}}} \exp \left[-\frac{\left(x-\mu_{\mathcal{I}}\right)^{2}}{2 \sigma_{\mathcal{I}}^{2}}\right],
$$

and

$$
p(x, y)=\frac{\exp \left[-\frac{\left(x-\mu_{\mathcal{I}}\right)^{2}+\left(y-\mu_{\mathcal{I}}\right)^{2}-2 \rho\left(x-\mu_{\mathcal{I}}\right)\left(y-\mu_{\mathcal{I}}\right)}{2 \sigma_{\mathcal{I}}^{2}\left(1-\rho^{2}\right)}\right]}{\sqrt{2 \pi\left(1-\rho^{2}\right)} \sigma_{\mathcal{I}}} .
$$

In what follows, we calculate $\phi_{\mathcal{I}}\left(I_{\mathrm{th}}\right)=\int_{I_{\mathrm{th}}}^{\infty} p(x) d x$ and $\varphi_{\mathcal{I}}\left(I_{\mathrm{th}}\right)=\iint_{I_{\mathrm{th}}}^{\infty} p(x, y) d x d y$ for the cases of $I_{\mathrm{th}} \geq \mu_{\mathcal{I}}$ and $I_{\text {th }}<\mu_{\mathcal{I}}$.

\section{A. The Case of $I_{\mathrm{th}} \geq \mu_{\mathcal{I}}$}

According to (4.2) [25] we obtain

$$
\phi_{\mathcal{I}}\left(I_{\mathrm{th}}\right) \stackrel{I_{\mathrm{th}} \stackrel{\geqq}{=} \mu_{\mathcal{I}}}{Q}\left(\tilde{I}_{\mathrm{th}}\right)=\frac{1}{\pi} \int_{0}^{\frac{\pi}{2}} \exp \left(-\frac{\tilde{I}_{\mathrm{th}}^{2}}{2 \sin ^{2} \theta}\right) d \theta,
$$

where $\tilde{I}_{\mathrm{th}}=\frac{I_{\mathrm{th}}-\mu_{\mathcal{I}}}{\sigma_{\mathcal{I}}}$. Similarly, using (4.18) [25] and the following equality

$$
\arctan \left(\sqrt{\frac{1+\rho}{1-\rho}}\right)=\frac{\pi}{4}+\frac{\arcsin (\rho)}{2},
$$

we obtain

$$
\begin{aligned}
\varphi_{\mathcal{I}}\left(I_{\mathrm{th}}\right) & \stackrel{I_{\mathrm{th}} \geqq \mu_{\mathcal{I}}}{=} Q\left(\tilde{I}_{\mathrm{th}}, \tilde{I}_{\mathrm{th}} ; \rho\right), \\
& \stackrel{(97)}{=} \frac{1}{\pi} \int_{0}^{\frac{\pi}{4}+\frac{\arcsin (\rho)}{2}} \exp \left(-\frac{\tilde{I}_{\mathrm{th}}^{2}}{2 \sin ^{2} \theta}\right) d \theta .
\end{aligned}
$$

Substitution of (96) and (98) into (25) results in (52). Moreover, $F_{\mathcal{I}}\left(I_{\text {th }}\right)=\int_{-\infty}^{I_{\mathrm{th}}} p(x) d x=1-Q\left(\tilde{I}_{\mathrm{th}}\right)$. By plugging $F_{\mathcal{I}}\left(I_{\mathrm{th}}\right)$ and (52) into (31) we obtain (53). 


\section{B. The Case of $I_{\mathrm{th}}<\mu_{\mathcal{I}}$}

In this case, the normalized threshold $\tilde{I}_{\text {th }}$ is less than 0. The integration region in the definition of $\phi_{\mathcal{I}}\left(I_{\mathrm{th}}\right)$ is $\left\{x \mid x \geq \tilde{I}_{\mathrm{th}}\right\}$, and the complementary region is $\left\{x \mid x<\tilde{I}_{\mathrm{th}}\right\}$, where the integral is equal to the integral over $\left\{x|x>| \tilde{I}_{\mathrm{th}} \mid\right\}$, given by (96), due to the symmetry of the Gaussian PDF. The integral on the whole region is 1 . Therefore, $\phi_{\mathcal{I}}\left(I_{\mathrm{th}}\right)=1-Q\left(\left|\tilde{I}_{\mathrm{th}}\right|\right)$, as written below

$$
\phi_{\mathcal{I}}\left(I_{\mathrm{th}}\right) \stackrel{I_{\mathrm{th}} \leq \mu_{\mathcal{I}}}{=} 1-\frac{1}{\pi} \int_{0}^{\frac{\pi}{2}} \exp \left(-\frac{\tilde{I}_{\mathrm{th}}^{2}}{2 \sin ^{2} \theta}\right) d \theta .
$$

For $\phi_{\mathcal{I}}\left(I_{\mathrm{th}}\right)$, its integral region is $U_{0}=$ $\left\{(x, y) \mid x \geq \tilde{I}_{\mathrm{th}}, y \geq \tilde{I}_{\mathrm{th}}\right\}$. Define the integral regions $U_{1}=\left\{(x, y) \mid x<\tilde{I}_{\text {th }}\right\}$ and $U_{2}=\left\{(x, y) \mid y<\tilde{I}_{\text {th }}\right\}$. The intersection of $U_{1}$ and $U_{2}$ is $U_{3}=U_{1} \cap U_{2}=$ $\left\{(x, y) \mid x<\tilde{I}_{\mathrm{th}}, y<\tilde{I}_{\mathrm{th}}\right\}$. Then the whole 2-D integral region is $U=\left(U_{0} \cup U_{1} \cup U_{2}\right) \backslash U_{3}$ with $\iint_{U} p(x, y) d x d y=1$. The integral over $U_{1}$ or $U_{2}$ is $Q\left(\left|\tilde{I}_{\mathrm{th}}\right|\right)$, and given by (96). The integral over $U_{3}$ is the same as that over $\left\{(x, y)|x>| \tilde{I}_{\mathrm{th}}|, y>| \tilde{I}_{\mathrm{th}} \mid\right\}$, due to the symmetry of the bivariate Gaussian PDF. The integral is $Q\left(\left|\tilde{I}_{\mathrm{th}}\right|,\left|\tilde{I}_{\mathrm{th}}\right| ; \rho\right)$, given by (98). Therefore,

$$
\begin{aligned}
\varphi_{\mathcal{I}}\left(I_{\mathrm{th}}\right) \stackrel{I_{\mathrm{th}}<\mu_{\mathcal{I}}}{=} 1-\left[2 Q\left(\left|\tilde{I}_{\mathrm{th}}\right|\right)-Q\left(\left|\tilde{I}_{\mathrm{th}}\right|,\left|\tilde{I}_{\mathrm{th}}\right| ; \rho\right)\right], \\
=1-\frac{2}{\pi} \int_{0}^{\frac{\pi}{2}} \exp \left(-\frac{\tilde{I}_{\mathrm{th}}^{2}}{2 \sin ^{2} \theta}\right) d \theta \\
+\frac{1}{\pi} \int_{0}^{\frac{\pi}{4}+\frac{\arcsin (\rho)}{2}} \exp \left(-\frac{\tilde{I}_{\mathrm{th}}^{2}}{2 \sin ^{2} \theta}\right) d \theta
\end{aligned}
$$

We obtain (52) by substituting (99) and (100) into (25). Similarly, we get $(53)$ easily by plugging $F_{\mathcal{I}}\left(I_{\text {th }}\right)$ and $(52)$ into (31).

\section{ACKNOWLEDGMENT}

Authors would like to thank the editor and the anonymous reviewers for their useful comments and suggestions.

\section{REFERENCES}

[1] G. J. Foschini and M. J. Gans, "On limits of wireless communications in a fading environment when using multiple antennas," Wireless Personal Commun., vol. 6, pp. 311-335, 1998.

[2] İ. E. Telatar, "Capacity of multi-antenna Gaussian channels," European Trans. Telecommun., vol. 10, pp. 585-595, 1999.

[3] D. W. Bliss, A. M. Chan, and N. B. Chang, "MIMO wireless communication channel phenomenology," IEEE Trans. Antennas Propag., vol. 52 , pp. 2073-2082, Aug. 2004

[4] M. T. Ivrlač, W. Utschick, and J. A. Nossek, "Fading correlations in wireless MIMO communication systems," IEEE J. Sel. Areas Commun., vol. 21, pp. 819-828, Jun. 2004.

[5] J. Maurer, C. Waldschmidt, T. Kayser, and W. Wiesbeck, "Characterisation of the time-dependent urban MIMO channel in FDD communication systems," in Proc. IEEE Veh. Technol. Conf., Jeju, Korea, 2003, pp. 544548.

[6] G. Lebrun, J. Gao, and M. Faulkner, "MIMO transmission over a timevarying channel using SVD," IEEE Trans. Wireless Commun., vol. 4, pp. 757-764, Mar. 2005.
[7] H. Sampath, P. Stoica, and A. Paulraj, "Generalized linear precoder and decoder design for MIMO channels using the weighted MMSE criterion," IEEE Trans. Commun., vol. 49, pp. 2198-2206, Dec. 2001.

[8] S. K. Jayaweera and H. V. Poor, "Capacity of multiple-antenna systems with both receiver and transmitter channel state information," IEEE Trans. Inf. Theory, vol. 49, pp. 2697-2709, Oct. 2003.

[9] D. Tse and P. Viswanath, Fundamentals of Wireless Communication. Cambridge, UK: Cambridge University Press, 2005.

[10] W. C. Jakes, Ed., Microwave Mobile Communications. New York: IEEE Press, 1994.

[11] A. Abdi, K. Wills, H. A. Barger, M. S. Alouini, and M. Kaveh, "Comparison of the level crossing rate and average fade duration of Rayleigh, Rice, and Nakagami fading models with mobile channel data," in Proc. IEEE Veh. Technol. Conf., Boston, MA, 2000, pp. 1850-1857.

[12] N. Youssef, T. Munakata, and M. Takeda, "Fade statistics in Nakagam fading environments," in Proc. IEEE Int. Symp. Spread Spec. Tech. App., Mainz, Germany, 1996, pp. 1244-1247.

[13] A. Abdi, J. A. Barger, and M. Kaveh, "A parametric model for the distribution of the angle of arrival and the associated correlation function and power spectrum at the mobile station," IEEE Trans. Veh. Technol., vol. 51, pp. 425-434, May 2002.

[14] A. Abdi, W. C. Lau, M. S. Alouini, and M. Kaveh, "A new simple model for land mobile satellite channels: First- and second-order statistics," IEEE Trans. Wireless Commun., vol. 2, pp. 519-528, May 2003.

[15] L. H. Ozarow, S. Shamai, and A. D. Wyner, "Information theoretic considerations for cellular mobile radio," IEEE Trans. Veh. Technol., vol. 43, pp. 359-378, May 1994.

[16] B. M. Hochwald, T. L. Marzetta, and V. Tarokh, "Multiple-antenna channel hardening and its implications for rate feedback and scheduling," IEEE Trans. Inf. Theory, vol. 50, pp. 1893-1909, Sep. 2004.

[17] Z. Wang and G. B. Giannakis, "Outage mutual information of spacetime MIMO channels," IEEE Trans. Inf. Theory, vol. 50, pp. 657-662, Apr. 2004.

[18] P. Smith and M. Shafi, "An approximate capacity distribution for MIMO systems," IEEE Trans. Commun., vol. 52, pp. 887-890, Jun. 2004.

[19] S. Wang and A. Abdi, "On the second-order statistics of the instantaneous mutual information of time-varying fading channels," in Proc. IEEE Int. Workshop Signal Processing Advances in Wireless Communications, New York, 2005, pp. 405-409.

[20] A. Giorgetti, M. Chiani, M. Shafi, and P. J. Smith, "Level crossing rates and MIMO capacity fades: impacts of spatial/temporal channel correlation," in Proc. IEEE Int. Conf. Commun., Anchorage, AK, 2003, pp. 3046-3050

[21] N. Zhang and B. Vojcic, "Evaluating the temporal correlation of MIMO channel capacities," in Proc. IEEE Global Telecommun. Conf., St. Louis, MO, 2005, pp. 2817-2821.

[22] S. Wang and A. Abdi, "Joint singular value distribution of two correlated rectangular complex Gaussian matrices and its application," SIAM J. Matrix Anal. \& Appl., vol. 29, pp. 972-981, Oct. 2007.

[23] I. S. Gradshteyn, I. M. Ryzhik, and A. Jeffrey, Eds., Table of Integrals, Series, and Products, 5th ed. San Diego, CA: Academic, 1994.

[24] M. K. Simon, Probability Distributions Involving Gaussian Random Variables: A Handbook for Engineers and Scientists. Boston, MA: Kluwer, 2002.

[25] M. K. Simon and M.-S. Alouini, Digital Communication over Fading Channels, 2nd ed. New York: Wiley-IEEE Press, 2004.

[26] S. Wang, "Envelope correlation coefficient for logarithmic diversity receivers revisited," IEEE Trans. Commun., vol. 55, pp. 2042-2046, Nov. 2007.

[27] W. B. Davenport and W. L. Root, An Introduction to the Theory of Random Signals and Noise. New York: Wiley, 1987.

[28] B. Kedem, Time Series Analysis by Higher Order Crossings. New York: IEEE Press, 1994.

[29] S. Wang, "MIMO fading channels: Models, statistics, and lowcomplexity estimators," Ph.D. dissertation, New Jersey Institute of Technology, Newark, NJ, 2006.

[30] A. T. James, "Distributions of matrix variates and latent roots derived from normal samples," Ann. Math. Statist., vol. 35, pp. 475-501, Jun. 1964.

[31] K. Acolatse and A. Abdi, "Efficient simulation of space-time correlated MIMO mobile fading channels," in Proc. IEEE Veh. Technol. Conf., Orlando, FL, 2003, pp. 652-656.

[32] A. J. Goldsmith and S.-G. Chua, "Adaptive coded modulation for fading channels," IEEE Trans. Commun., vol. 46, pp. 595-602, May 1998.

[33] P. Beckmann, Orthogonal Polynomials for Engineers and Physicists. Boulder, CO: Golem Press, 1973. 
[34] V. S. Adamchik and O. I. Marichev, "The algorithm for calculating integrals of hypergeometric type functions and its realization in REDUCE system," in Proc. Int. Conf. Symbolic Algebraic Computation, Tokyo, Japan, 1990, pp. 212-224.

[35] А. П. Прудников, Ю. А. Брычков, and О. И. Маричев, Интегралы и ряды. В 3 т. Том. 3. Специальные функции. Дополнительные главы., 2nd ed. MOCKВА: ФИЗМАТЛИТ, 2003.

[36] K. O. Geddes, M. L. Glasser, R. A. Moore, and T. C. Scott, "Evaluation of classes of definite integrals involving elementary functions via differentiation of special functions," Applicable Algebra In Engineering, Communication And Computing, vol. 1, pp. 149-165, 1990.

[37] N. R. Goodman, "The distribution of the determinant of a complex Wishart distributed matrix," Ann. Math. Statist., vol. 34, pp. 178-180, Mar. 1963.

[38] R. L. Graham, D. E. Knuth, and O. Patashnik, Eds., Concrete Mathematics: A Foundation for Computer Science, 2nd ed. Boston, MA: Addison-Wesley, 1994.

[39] A. K. Gupta and D. K. Nagar, Matrix Variate Distributions. New York: Chapman \& Hall/CRC, 1999.

[40] А. П. Прудников, Ю. А. Брычков, and О. И. Маричев, Интегралы и ряды. Специальные функции. МОСКВА: Наука, 1983.

Shuangquan Wang received his B.S. degree in Computer Engineering from Xi'an Institute of Posts and Telecommunications, Xi'an, China, in 1998, M.S. degree in Electrical Engineering from Beijing University of Posts and Telecommunications (BUPT), Beijing, China, in 2001, and Ph.D. degree in Electrical Engineering from New Jersey Institute of Technology, Newark, USA, in 2006. From 2001 to 2002, he worked on interference and noise power estimation and multi-user receiver design in TD-SCDMA systems in Siemens Ltd. China, Beijing. From 2006 to 2007, he worked in NEC Laboratories America, Inc., Princeton, where his research topics included low-complexity LLR computation for high-order QAM and PSK constellations, efficient ML MIMO receiver design, PCI and CQI calculation in LTE systems. Now he is with Broadcom Corp., Matawan, and works on HSPA+. His research interests include MIMO channel modeling, estimation and its second-order statistics, random matrix theory and its application in wireless MIMO communications, multiuser communications, and space-time transceiver design in MIMOOFDM systems.

Dr. Wang has several granted and pending patents, and won several awards for his research contributions. These include the BUPT EI index prize in 2005, the 2005 New Jersey Inventors Hall of Fame Graduate Student Award, the First Place in Graduate Poster Contest, IEEE GLOBECOM'05 and the 2007 NJIT Hashimoto Prize.

Ali Abdi (S'98, M'01, SM'06) received the Ph.D. degree in electrical engineering from the University of Minnesota, Minneapolis. He joined the Department of Electrical and Computer Engineering of New Jersey Institute of Technology (NJIT), Newark, where he is currently an Associate Professor. His current research interests include characterization and estimation of wireless channels, digital communication in underwater and terrestrial channels, blind modulation recognition, systems biology, molecular networks and cell signaling. He is a member of Society for Neuroscience. Dr. Abdi was an Associate Editor for IEEE Transactions on Vehicular Technology from 2002 to 2007. His recent professional activities include co-chairing the Communication and Information Theory Track of the 2008 IEEE ICCCN (International Conference on Computer Communications and Networks), and co-organizing/co-chairing a special session entitled "Acoustic Particle Velocity and Vector Fields: Signal Processing and Communication Applications," for the 159th Meeting of the Acoustical Society of America in 2010. He has several granted and pending patents and his recent awards include 2008 New Jersey Inventors Hall of Fame (NJIHoF) Innovators Award and 2009 IEEE Region 1 Award for outstanding leadership and contributions in underwater communication, $2009 \mathrm{NJIHoF}$ Innovators Award for a novel cross-disciplinary engineering-biology invention and 2006 NJIT Excellence in Teaching Award, in the category of Excellence in Team, Interdepartmental, Multidisciplinary, or Non-Traditional Teaching. 